TRANSACTIONS OF THE

AMERICAN MATHEMATICAL SOCIETY

Volume 356, Number 3, Pages 1233-1270

S 0002-9947(03)03412-3

Article electronically published on October 6, 2003

\title{
UNIVERSAL COVERS FOR HAUSDORFF LIMITS OF NONCOMPACT SPACES
}

\author{
CHRISTINA SORMANI AND GUOFANG WEI
}

\begin{abstract}
We prove that if $Y$ is the Gromov-Hausdorff limit of a sequence of complete manifolds, $M_{i}^{n}$, with a uniform lower bound on Ricci curvature, then $Y$ has a universal cover.
\end{abstract}

\section{INTRODUCTION}

One of the main trends in Riemannian geometry today is the study of Gromov Hausdorff limits. The starting point is Gromov's precompactness theorem. Namely a sequence of complete Riemannian $n$-manifolds with a uniform lower bound on their Ricci curvatures have a converging subsequence. Moreover the limit space is a complete length space. That is, it is a metric space such that between every two points there is a length minimizing curve whose length is the distance between the two points. See $[\mathrm{Gr}]$; also $[\mathrm{BBI}]$. To prove this theorem, the only property of Ricci curvature that Gromov uses is the Bishop-Gromov volume comparison theorem $\mathrm{BiCr}$, $\mathrm{Gr}$ which provides an estimate on the number of disjoint small balls which fit in a large ball.

When the sectional curvature of the sequence is uniformly bounded from below, the limit space is well understood. Namely, it is an Alexandrov space with curvature bounded below and, by the work of Perelman [Pl1], it is a stratified topological manifold that is locally contractible.

In the case when only Ricci curvature is bounded from below Menguy has shown that the limit space can have infinite topological type on arbitrarily small balls even with the additional assumptions of a uniform positive lower bound on the volume and nonnegative Ricci curvature on the $M_{i}^{n}$ [Me. This is based on an example of Perelman $[\mathrm{Pl} 2$. In the positive direction Cheeger and Colding [ChCo1 $-\mathrm{ChCo} 4$ have proven a number of breakthrough results regarding the regularity and geometric properties of the limit spaces including the construction of a measure which satisfies the volume comparison theorem. Of course one cannot expect regularity in a $C^{1, \alpha}$ sense but rather a statement regarding the tangent cones at regular points. This should be contrasted with Anderson's theorem [An1] that $Y$ is a $C^{1, \alpha}$ manifold when a uniform injectivity radius and two-sided Ricci bounds are imposed on the sequence. Despite these positive results these limit spaces are not yet completely understood and many questions remain.

Received by the editors July 24, 2002 and, in revised form, February 28, 2003.

2000 Mathematics Subject Classification. Primary 53C20.

The first author was partially supported by NSF Grant \# DMS-0102279 and a grant from The City University of New York PSC-CUNY Research Award Program.

The second author was partially supported by NSF Grant \# DMS-9971833. 
In this paper we ask whether the limit space has a universal cover (see Definition (2.2). Note that without the Ricci bound, a limit space may not have a universal cover. The Hawaii Ring (Example 2.1) is a limit of compact manifolds with no curvature bound but increasingly large fundamental groups (see SoWei Example 2.7]). In contrast authors proved that the limit spaces of sequences of simply connected uniformly bounded compact length spaces have universal covers which are the spaces themselves SoWei Thm 1.5]. With a Ricci curvature lower bound we answer the question affirmatively without any assumption on simple connectivity. Namely we prove the following theorem:

Theorem 1.1. If $(Y, p)$ is the pointed Gromov-Hausdorff limit of a sequence of $n$-dimensional complete Riemannian manifolds $\left(M_{i}^{n}, p_{i}\right)$ with Ric $\geq(n-1) H$, then the universal cover of $Y$ exists.

In their previous article, the authors proved this existence theorem with an additional assumption that the manifolds in the sequence were compact with a uniform upper bound on the diameter [SoWei, Theorem 1.1]. To prove that result, we defined $\delta$-covers, covers which unravel holes of a size greater than $\delta$ (see Definition 2.5). We showed that when $\delta$ is the injectivity radius of a manifold, the $\delta$-cover is the universal cover of that manifold. These $\delta$-covers were complete manifolds with the same lower bound on the Ricci curvature as the original manifold, so we were able to apply Gromov's precompactness theorem and Cheeger-Colding's renormalized limit measures to study their limits. We then proved that for a compact length space that has a universal cover, the universal cover is always some $\delta$-cover SoWei. Proposition 3.2]. Ultimately we showed that there was a $\delta$ sufficiently small such that the limit of the $\delta$-covers was the universal cover of the limit space of the original sequence.

We cannot hope for such a strong statement in the noncompact case. First, even for a complete manifold the universal cover may not be any $\delta$-cover. This can be seen from Nabonnand's example, which is a complete manifold with positive Ricci curvature that is not simply connected and yet it is its own $\delta$-cover for all values of $\delta[\mathrm{Nab}]$. It has a loop which is homotopic to a sequence of increasingly small loops that diverge to the infinity of the manifold. See also Wei]. Secondly, the universal cover of the limit may not come from any cover of the sequence as the following example shows. That is, pointed limits of complete noncompact simply connected manifolds with nonnegative sectional curvature can converge to a cylinder.

Example 1.2. If we take $M^{2}$, the half cylinder capped off by a hemisphere (and suitably smoothed, which is simply connected and has sectional curvature $\geq 0$ ), and $p_{i}$ a sequence of points in $M$ going to infinity, then $\left(M^{2}, p_{i}\right)$ converges to a long cylinder. Its universal cover $\left(\mathbb{R}^{2}\right)$ cannot come from the limit of any cover of $M^{2}$ since its only cover is $M^{2}$ itself.

To avoid these problems, it is natural to work locally since pointed GromovHausdorff convergence is defined locally. So we will consider covers of balls. To study the fundamental group we have to use the intrinsic length space metric on the balls rather than the restricted metric so we can measure the lengths of representatives of the fundamental group. However, there has been some inconsistency in the literature as to whether manifolds which converge in the pointed GromovHausdorff sense have Gromov-Hausdorff convergence of their balls with respect to the intrinsic as well as the restricted metrics. This is clarified in our Appendix, 
allowing us to use the intrinsic metric as long as the radii of the balls vary in a prescribed way.

Even so, we will not use the $\delta$-covers of balls of the converging sequence because it is not clear if the sequences of such $\delta$-covers have any converging subsequences. In general the fundmental group of a ball in $M$ may have exponential growth even if $M$ has Ric $\geq 0$ and is compact.

Example 1.3. $M^{2}$ is a 2-torus obtained by gluing the sides of a 2-by-2 square. Take a ball at the center with radius $1<r<\sqrt{2}$; then the ball is homotopic to the figure eight and its fundamental group has exponential growth.

In fact, in Example 3.2 we give a sequence of smooth 2-dimensional manifolds $M_{k}$ with nonnegative sectional curvature such that the universal cover of the balls of radius 1 in $M_{k}$ have no converging subsequence. The reason that Gromov's precompactness theorem fails for these covers is that they are manifolds with boundary and the order of their volume growth diverges to infinity.

Thus, in Section 2, we introduce the relative delta cover (see Definition 2.6): the connected lift of a ball of small radius inside the delta cover of a concentric ball of a larger radius. We show that if relative delta covers converge, then the limit is almost the relative delta cover of balls in the limit (Theorem 2.9). With a uniform Ricci curvature lower bound, we can control the volumes in a relative delta cover on the scale of the larger ball. We can then bound the number of generators of the deck transformations of the relative delta cover and, in turn, use this to control the volume growth of the relative delta cover, proving that relative delta covers satisfy the Gromov precompactness theorem even though they are manifolds with boundary with large volume growth. This is done in Section 3 See Proposition 3.1

In Subsection 3.2 we extend Cheeger-Colding's construction of a renormalized limit measure to these limit spaces although it must be warned that these measures only satisfy the Bishop-Gromov volume comparison theorem locally.

One might hope to construct the universal cover of the limit space of the original manifold by piecing together the limits of relative delta covers where the delta varies from piece to piece. However it is possible that these relative delta covers are nontrivial covers of balls in the universal cover no matter how small we choose delta and how large we choose the ball. See Example 4.1 Instead we use Theorem 2.5 from Section 2, which relates the stability of the relative delta covers with the existence of the universal cover. We use the renormalized limit measure to obtain the stability of the relative delta covers. This completes the proof of Theorem 1.1

In Section 4 we study the properties of the universal cover and give some applications. First we prove that regions in the universal cover are limits of relative delta covers with changing delta and $R \rightarrow \infty$. See Theorem 4.2 and Corollary 4.3 Using this we are able to show that the global Bishop-Gromov volume comparison does hold for the lifted renormalized limit measure on the univeral cover of the limit space (Theorem 4.5).

We also prove the splitting theorem on the universal cover of the limit space when the $M_{i}$ have Ricci $\geq-(n-1) \epsilon_{i}$ with $\epsilon_{i} \rightarrow 0$ [Theorem 4.6] using Cheeger-Colding's almost splitting theorem.

These two theorems allow us to generalize various results of Milnor, Anderson and Sormani to limit spaces $Y$; see Corollaries 4.7 4.8 and 4.9 
The authors would like to thank Professor D. Cooper for providing us with Example 4.1. We would also like to thank Professor J. Cheeger for emails concerning the details of the construction of the renormalized limit measure in [ChCo2].

\section{UNIVERSAL COVER AND $\delta$-COVERING SPACES}

2.1. Covers and universal covers. First we recall some basic definitions.

Definition 2.1. We say $\bar{X}$ is a covering space of $X$ if there is a continuous map $\pi: \bar{X} \rightarrow X$ such that $\forall x \in X$ there is an open neighborhood $U$ such that $\pi^{-1}(U)$ is a disjoint union of open subsets of $\bar{X}$, each of which is mapped homeomorphically onto $U$ by $\pi$ (we say $U$ is evenly covered by $\pi$ ).

Definition 2.2 ([Sp pp. 62,83$]$ ). We say $\tilde{X}$ is a universal cover of $X$ if $\tilde{X}$ is a cover of $X$ such that for any other cover $\bar{X}$ of $X$, there is a commutative triangle formed by a continuous map $f: \tilde{X} \rightarrow \bar{X}$ and the two covering projections.

Example 2.1. The Hawaii Ring (see for example Spanier $[\mathrm{Sp}]$ ) is a length space which consists of an infinite set of rings of radii decreasing to 0 that are all joined at a common point. This ring has no universal cover because the universal cover would have to have an isometric lift of a ball about this common point. Thus the possible universal cover would contain many closed rings itself and would therefore have a nontrivial cover.

Let $\mathcal{U}$ be any open covering of $Y$. For any $p \in Y$, by [Sp, Page 81], there is a covering space, $\tilde{Y}_{\mathcal{U}}$, of $Y$ with covering group $\pi_{1}(Y, \mathcal{U}, p)$, where $\pi_{1}(Y, \mathcal{U}, p)$ is a normal subgroup of $\pi_{1}(Y, p)$, generated by homotopy classes of closed paths having a representative of the form $\alpha^{-1} \circ \beta \circ \alpha$, where $\beta$ is a closed path lying in some element of $\mathcal{U}$ and $\alpha$ is a path from $p$ to $\beta(0)$.

Recall a few facts about covering spaces constructed from $\mathcal{U}$ [Sp, Page 81]. If $\mathcal{V}$ is an open covering of $Y$ that refines $\mathcal{U}$, then $\pi_{1}(Y, \mathcal{V}, p) \subset \pi_{1}(Y, \mathcal{U}, p)$, or $\tilde{Y}_{\mathcal{V}}$ covers $\tilde{Y}_{\mathcal{U}}$. If $\pi: \bar{Y} \rightarrow Y$ is a covering projection and $\mathcal{U}$ is an open covering of $Y$ such that each of its open sets is evenly covered by $\pi$, then $\tilde{Y}_{\mathcal{U}}$ covers $\bar{Y}$.

Recall also that given a metric space $(X, d)$, there is an induced length metric, $d_{l}$ [Gr, Page 2] (see also [BBI]), given by

$$
d_{l}(x, y)=\inf _{f}\{L(f) \mid f:[0,1] \rightarrow X \text { is a continuous map with } f(0)=x, f(1)=y\},
$$

where

$$
L(f)=\sup \sum_{i=0}^{n} d\left(f\left(t_{i}\right), f\left(t_{i+1}\right)\right)
$$

and the sup is taken among all finite partitions of $[0,1], 0=t_{0} \leq t_{1} \leq \cdots \leq t_{n+1}=$ 1. In general $d_{l} \geq d$.

Definition 2.3. A metric space $(X, d)$ is called a length space (or path metric space as in $[\mathrm{Gr}]$ ) if $d=d_{l}$.

Definition 2.4. Given a length space $(X, d)$ and a covering map $\pi: \bar{X} \rightarrow X$, where $\bar{X}$ is equipped with natural lifted length metric $\bar{d}$, the Dirichlet fundamental domain of $X$ about $\bar{x}$, for any $\bar{x} \in \bar{X}$, is defined:

$$
F D_{\bar{x}}=\{\bar{q} \in \bar{X}: \bar{d}(\bar{x}, \bar{q}) \leq \bar{d}(\bar{z}, \bar{q}) \forall \bar{z} \in \bar{X} \text { s.t. } \pi(\bar{z})=\pi(\bar{x})\} .
$$


If $Y$ is a length space with metric $d_{Y}$ and $X \subset Y$, then we will use $d_{Y}$ to denote the restricted metric of $Y$ on $X$ and $d_{X}$ to denote the induced length metric or intrinsic metric on $X$.

Let $X$ be a length space, denote $B(p, R)$ a closed ball in $X$, i.e. $B(p, R)=\{x \in$ $\left.X \mid d_{X}(x, p) \leq R\right\}$, and $B_{p}(s)$ an open ball measured in various metrics which will be stated on each occasion. Observe that:

Lemma 2.2. The restricted metric on $B(p, R)$ from $X$ is the same as the intrinsic metric on $B(p, 2 R+\epsilon)$ restricted to $B(p, R)$ for any $\epsilon>0$. Namely,

$$
d_{X}\left(q_{1}, q_{2}\right)=d_{B(p, 2 R+\epsilon)}\left(q_{1}, q_{2}\right), \quad \forall q_{1}, q_{2} \in B(p, R) .
$$

Now let us recall the $\delta$-covers we introduced in SoWei].

Definition 2.5. Given $\delta>0$, the $\delta$-cover, denoted $\tilde{Y}^{\delta}$, of a length space $Y$ is defined to be $\tilde{Y}_{\mathcal{U}_{\delta}}$, where $\mathcal{U}_{\delta}$ is the open covering of $Y$ consisting of all balls of radius $\delta$.

The covering group will be denoted $\pi_{1}(Y, \delta, p) \subset \pi_{1}(Y, p)$ and the group of deck transformations of $\tilde{Y}^{\delta}$ will be denoted $G(Y, \delta)=\pi_{1}(Y, p) / \pi_{1}(Y, \delta, p)$.

It is easy to see that a delta cover is a regular or Galois cover. That is, the lift of any closed loop in $\mathrm{Y}$ is either always closed or always open in its delta cover.

Note that $\tilde{Y}^{\delta_{1}}$ covers $\tilde{Y}^{\delta_{2}}$ when $\delta_{1} \leq \delta_{2}$.

In SoWei, Prop. 3.2] we proved that if a compact length space $Y$ has a universal cover, then it is a delta cover. In fact $Y$ has a universal cover iff the delta covers stabilize: there exists a $\delta_{0}>0$ such that $\tilde{Y}^{\delta}=\tilde{Y}^{\delta_{0}}$ for all $\delta<\delta_{0}$ [SoWei, Thm 3.7].

However, this is not true for a noncompact length space. For example, a cylinder with two cusped ends has $\mathbb{R}^{2}$ as a universal cover but all of its delta covers are trivial. Thus we work locally. Since the $\delta$ covers of balls, $\tilde{B}(p, r)^{\delta}$, and their covering groups, $G(B(p, r), \delta)$, are not well controlled in the Gromov-Hausdorff sense as discussed in the Introduction, we define a relative $\delta$ cover:

Definition 2.6. Given closed balls $B(p, r) \subset B(p, R) \subset Y$ with intrinsic metrics, where $r<R$, let $\tilde{B}(p, r)^{\delta}$ and $\tilde{B}(p, R)^{\delta}$ be their $\delta$-covers. The relative $\delta$-cover, denoted $\tilde{B}(p, r, R)^{\delta}$, is a connected lift of $B(p, r)$ to $\tilde{B}(p, R)^{\delta}$.

Clearly relative $\delta$ covers are also regular. The group of deck transformations of $\tilde{B}(p, r, R)^{\delta}$ is denoted $G(p, r, R, \delta)$ and is, in fact, the image of $i_{*}: G(B(p, r), \delta) \rightarrow$ $G(B(p, R), \delta)$. So we have the covering relation $\tilde{B}(p, r)^{\delta} \rightarrow \tilde{B}(p, r, R)^{\delta} \rightarrow B(p, r)$.

Lemma 2.3. The covering map

$$
\pi^{\delta}(r, R):\left(\tilde{B}(p, r, R)^{\delta}, d_{\tilde{B}(p, r, R)^{\delta}}\right) \rightarrow\left(B(p, r), d_{B(p, r)}\right)
$$

is an isometry on balls of radius $\delta / 3$.

Proof. If $\tilde{x} \in \tilde{B}(p, r, R)^{\delta}$, the intrinsic metric's ball $B_{\tilde{x}}(s), d_{\tilde{B}(p, r, R)^{\delta}}$ is a subset of the restricted ball $B_{\tilde{x}}(s), d_{\tilde{B}(p, R)^{\delta}}$. Thus, for all $s<\delta$ it is mapped homeomorphically onto its image, $U_{x}(s)$, in $B(p, r)$ under the map $\pi^{\delta}(r, R)$ (which agrees with $\pi^{\delta}(R)$ as a homeomorphism).

So if $q_{1}, q_{2} \in U_{x}(\delta / 3)$, they lift to unique $\tilde{q}_{1}, \tilde{q}_{2} \in\left(B_{\tilde{x}}(\delta / 3), d_{\tilde{B}(p, r, R)^{\delta}}\right)$. In fact the lifts are unique in $\left(B_{\tilde{x}}(\delta), d_{\tilde{B}(p, r, R)^{\delta}}\right)$. Furthermore, since $\pi^{\delta}(r, R)$ is distance decreasing,

$$
d_{B(p, r)}\left(q_{1}, q_{2}\right) \leq d_{\tilde{B}(p, r, R)^{\delta}}\left(\tilde{q}_{1}, \tilde{q}_{2}\right)<2(\delta / 3) .
$$


Thus there is a curve $C \subset B(p, r)$ joining $q_{1}$ to $q_{2}$ of length $L(C)=d_{B(p, r)}\left(q_{1}, q_{2}\right)$ $<2 \delta / 3$. The curve lifts to $\tilde{C} \subset \tilde{B}(p, r, R)^{\delta}$ starting from $\tilde{q}_{1}$ and remaining in $B_{\tilde{q}_{1}}(2 \delta / 3) \subset B_{\tilde{x}}(\delta), d_{\tilde{B}(p, r, R)^{\delta}}$. Since the end point of $\tilde{C}$ is a lift of $q_{2}$ and lifts are unique in $B_{\tilde{x}}(\delta), \tilde{C}$ joins $\tilde{q_{1}}$ to $\tilde{q}_{2}$. Thus

$$
d_{\tilde{B}(p, r, R)^{\delta}}\left(\tilde{q}_{1}, \tilde{q}_{2}\right)=L(\tilde{C})=L(C)=d_{B(p, r)}\left(q_{1}, q_{2}\right) .
$$

This implies that $U_{x}(\delta / 3)=\left(B_{x}(\delta / 3), d_{B(p, r)}\right)$ and $\pi^{\delta}(r, R)$ is an isometry on balls of radius $\delta / 3$.

These relative delta covers provide us with a means of constructing a universal cover.

Lemma 2.4. Let $Y$ be a length space. If for all $x \in Y$ there exists an open neighborhood of $x, U_{x}$, which lifts homeomorphically to all covers of $Y$, then the cover $\tilde{Y}_{\mathcal{U}}$ created using the open sets $U_{x}$ is the universal cover of $Y$.

Proof. Let $\pi: \bar{Y} \rightarrow Y$ be any covering projection. Then by the definition of $\mathcal{U}, \mathcal{U}$ is a covering of $Y$ by open sets each evenly covered by $\pi$. Thus, $\tilde{Y}_{\mathcal{U}}$ covers $\bar{Y}$.

Theorem 2.5. For a length space $Y$, its universal cover $\tilde{Y}$ exists if there is $y \in Y$ such that for all $r>0$, there exists $R \geq r$, such that $\tilde{B}(y, r, R)^{\delta}$ stabilizes for all $\delta$ sufficiently small.

Remark. Note there are $Y$ such that the universal cover of $Y$ exists but not for balls in $Y$. Such an example can be found in SoWei, Example 2.6]. This example is a compact length space which is its own universal cover but has balls that are homotopic to the Hawaii Ring. That is one reason we use relative delta covers instead of just delta covers of balls.

Proof. We will construct $\tilde{Y}$ using Lemma 2.4. For any $x \in Y$, there exists $r$ such that $x \in B_{y}(r / 10)$. Since $\tilde{B}(y, r, R)^{\delta}$ stabilizes, there exist $\delta_{r, R}>0$ depending on $r$ and $R$ and $\delta_{x}<r / 10$ depending on $x, r$ and $R$ such that $B_{x}\left(\delta_{x}\right)$ lifts homeomorphically to $\tilde{B}(y, r, R)^{\delta}$ for all $0<\delta<\delta_{0}$, where $\delta_{0}=\min \left\{\delta_{x}, \delta_{r, R}\right\}$. Note that by keeping $\delta_{x}<r / 10$ we avoid having to choose a metric and stay clear of the boundary.

Now $\tilde{B}(y, R)^{\delta}$ is just the disjoint union of some copies of the relative delta cover, $\tilde{B}(y, r, R)^{\delta}$, so $B_{x}\left(\delta_{x}\right)$ lifts homeomorphically to $\tilde{B}(y, R)^{\delta}$ as well.

Let $\pi: \bar{Y} \rightarrow Y$ be any cover of $Y$. Then any connected component $\bar{B}$ of $\pi^{-1}(B(y, R)) \subset \bar{Y}$ is a covering of $B(y, R)$. We need only show that $B_{x}\left(\delta_{x}\right)$ lifts homeomorphically to $\bar{B}$ and thus $\bar{Y}$.

Since $B(y, R)$ is compact, we can apply the proof of [SoWei, Thm 3.7] to say there exists a $\delta_{1}>0$ such that $\tilde{B}(y, R)^{\delta}$ covers $\bar{B}$ for all $\delta<\delta_{1}$. If we take $\delta<\min \left\{\delta_{1}, \delta_{0}\right\}$, then $B_{x}\left(\delta_{x}\right)$ lifts homeomorphically to $\tilde{B}(y, R)^{\delta}$. Thus it projects down homeomorphically to $\bar{B}$ as well. By Lemma 2.4 the universal cover of $Y$ exists.

2.2. Covers and convergence. Recall that $G(p, r, R, \delta)$ is the group of deck transforms of the relative $\delta$-cover $\tilde{B}(p, r, R)^{\delta}$ defined in Definition 2.6. Note that $G(p, r, R, \delta)$ can be represented as equivalence classes of loops based at $p$ in the small ball, $B(p, r)$, where $\gamma_{1}$ is equivalent to $\gamma_{2}$ if $\gamma_{2}^{-1} \circ \gamma_{1}$ is homotopic in the large ball, $B(p, R)$, to a loop composed of elements of the form $(\alpha * \beta) * \alpha^{-1}$, where $\beta$ is a closed path lying in a ball of radius $\delta$ and $\alpha$ is a path from $p$ to $\beta(0)$. 
Definition 2.7. For any $g \in G(p, r, R, \delta)$, we can define the (translative) $\delta$-length of $g$ by

$$
l(g, r, R, \delta)=\min _{q \in \tilde{B}(p, r, R)^{\delta}} d_{\tilde{B}(p, r, R)^{\delta}}(q, g(q)) .
$$

We have the following basic properties for $\delta$-length.

Lemma 2.6. For all nontrivial $g \in G(p, r, R, \delta)$, the $\delta$-length of $g$,

$$
l(g, r, R, \delta) \geq \delta .
$$

For all $\delta_{1} \leq \delta_{2}$ we have

$$
l\left(g, r, R, \delta_{1}\right) \geq l\left(g, r, R, \delta_{2}\right) .
$$

Proof. Since $G(p, r, R, \delta)$ is a subgroup of $G(B(p, R), \delta), g$ is also nontrivial in $G(B(p, R), \delta)$. By [SoWei, Lemma 3.1],

$$
l(g, R, \delta)=\min _{q \in \tilde{B}(p, R)} d_{\tilde{B}(p, R)}(q, g(q)) \geq \delta .
$$

Clearly $l(g, r, R, \delta) \geq l(g, R, \delta)$. So $l(g, r, R, \delta) \geq \delta$.

(2.8) follows from the fact that $\tilde{B}(p, r, R)^{\delta_{1}}$ covers $\tilde{B}(p, r, R)^{\delta_{2}}$ and it is distance nonincreasing.

We now study the relationship between the relative $\delta$-covers of two distinct balls which are close in the Gromov-Hausdorff sense, extending Theorem 3.4 in [SoWei]. There is some difficulty involving the use of restricted versus intrinsic length metrics when taking Gromov-Hausdorff approximations. So we ask the reader to refer to the Appendix, and, in particular, Definition 5.3 at this time.

Theorem 2.7. Let $B\left(p_{i}, r_{i}\right) \subset B\left(p_{i}, R_{i}\right) \subset Y_{i}, i=1,2$, be balls each with intrinsic metrics. If there is a pointed $\epsilon$-Hausdorff approximation $f: B\left(p_{1}, R_{1}\right) \rightarrow$ $B\left(p_{2}, R_{2}\right)$ such that its restriction also gives a pointed $\epsilon$-Hausdorff approximation from $B\left(p_{1}, r_{1}\right)$ to $B\left(p_{2}, r_{2}\right)$, then there is a surjective homomorphism, $\Phi$ : $G\left(p_{1}, r_{1}, R_{1}, \delta_{1}\right) \rightarrow G\left(p_{2}, r_{2}, R_{2}, \delta_{2}\right)$ for any $\delta_{1}>10 \epsilon$ and $\delta_{2}>\delta_{1}+10 \epsilon$.

Note that Theorem [2.7 combined with Corollary [5.2] of the Appendix gives us the following.

Corollary 2.8. Suppose $\left(M_{i}, p_{i}\right)$ converges to $(Y, y)$ in the pointed Gromov-Hausdorff topology. Then for any $r<R, \delta_{1}<\delta_{2}$, there exist sequences $r_{i} \rightarrow r$ and $R_{i} \rightarrow$ $R$ such that $B\left(p_{i}, r_{i}\right)$ and $B\left(p_{i}, R_{i}\right)$ converge to $B(p, r)$ and $B(p, R)$ with respect to intrinsic metrics, and a number $N$ sufficiently large depending upon $r, R, \delta_{2}$ and $\delta_{1}$ such that $\forall i \geq N$ there is a surjective map $\Phi_{i}: G\left(p_{i}, r_{i}, R_{i}, \delta_{1}\right) \rightarrow G\left(y, r, R, \delta_{2}\right)$.

Proof of Theorem 2.7. For a closed curve $\gamma:[0,1] \rightarrow B\left(p_{1}, R_{1}\right)$ with $\gamma(0)=\gamma(1)=$ $p_{1}$, construct a $5 \epsilon$-partition of $\gamma$ as follows. On $\Gamma:=\gamma([0,1])$ choose a partition $0=t_{0} \leq t_{1} \leq \cdots \leq t_{m}=1$ such that for $x_{i}=\gamma\left(t_{i}\right)$, one has $d\left(x_{i}, x_{i+1}\right)<5 \epsilon$ for $i=0, \cdots, m-1$. $\left\{x_{0}, \cdots, x_{m}\right\}$ is called a $5 \epsilon$-partition of $\gamma$.

For each $x_{i}$, we set $y_{m}=y_{0}=p_{2}$ and $y_{i}=f\left(x_{i}\right), i=1, \cdots, m-1$. If $y_{i}, y_{i+1}$ are both in $B\left(p_{2}, r_{2}\right)$, connect them by a minimal length curve, $\bar{\gamma}_{i}$ in $B\left(p_{2}, r_{2}\right)$; otherwise connect them with a minimal length curve, $\bar{\gamma}_{i}$ in $B\left(p_{2}, R_{2}\right)$. This yields a closed curve $\bar{\gamma}$ in $B\left(p_{2}, R_{2}\right)$ based at $p_{2}$ consisting of $m$ minimizing segments each having length $\leq 6 \epsilon$. This construction guarantees that if $\gamma \in B\left(p_{1}, r_{1}\right)$, then $\bar{\gamma} \in B\left(p_{2}, r_{2}\right)$. 
Any $\alpha \in G\left(p_{1}, r_{1}, R_{1}, \delta_{1}\right)$ can be represented by some rectifiable closed curve $\gamma$ in $B\left(p_{1}, r_{1}\right)$, so we can hope to define

$$
\Phi(\alpha)=\Phi([\gamma]):=[\bar{\gamma}] \in G\left(p_{2}, r_{2}, R_{2}, \delta_{2}\right) .
$$

First we need to verify that $\Phi$ does not depend on the choice of $\gamma$ such that $[\gamma]=\alpha$.

Using the fact that $18 \epsilon<\delta_{2}$, one easily sees that $[\bar{\gamma}]$ does not depend on the choice of minimizing curves $\bar{\gamma}_{i}$, nor on the special partition $\left\{x_{1}, \cdots, x_{m}\right\}$ of $\gamma([0,1])$. Moreover using additionally the uniform continuity of a homotopy one can see that $[\bar{\gamma}]$ only depends on the homotopy class of $\gamma$ in $\pi_{1}\left(B\left(p_{1}, r_{1}\right), p_{1}\right)$.

Thus it is also easy to check that $\Phi$ is a homomorphism from $\pi_{1}\left(B\left(p_{1}, r_{1}\right), p_{1}\right)$ to $G\left(p_{2}, r_{2}, R_{2}, \delta_{2}\right)$. However $\alpha \in G\left(p_{1}, r_{1}, R_{1}, \delta_{1}\right)$, not $\pi_{1}\left(B\left(p_{1}, r_{1}\right), p_{1}\right)$.

Suppose $\gamma_{1}$ and $\gamma_{2}$ are both representatives of $\alpha \in G\left(p_{1}, r_{1}, R_{1}, \delta_{1}\right)$. Then $\gamma_{1} * \gamma_{2}^{-1}$ is, in $B\left(p_{1}, R_{1}\right)$, homotopic to a loop $\gamma_{3}$ generated by loops of the form $\alpha * \beta * \alpha^{-1}$, where $\beta$ is a closed path lying in a ball of radius $\delta_{1}$ and $\alpha$ is a path from $p_{1}$ to $\beta(0)$. So $\left[\overline{\gamma_{1}}\right]=\left[\overline{\gamma_{3}}\right] *\left[\overline{\gamma_{2}}\right]$ and we need only show that $\left[\overline{\gamma_{3}}\right]$ is trivial in $G\left(p_{2}, r_{2}, R_{2}, \delta_{2}\right)$.

In fact $\overline{\gamma_{3}}$ can be chosen as follows. The $y_{i}$ 's corresponding to the $x_{i}$ 's from the $\beta$ segments of $\gamma_{3}$ are all within $\delta_{1}+\epsilon$ of a common point and the minimal geodesics between them are within $\delta_{1}+(1+6 / 2) \epsilon<\delta_{2}$. Furthermore, the $y_{i}$ 's corresponding to the $x_{i}$ 's from the $\alpha$ and $\alpha^{-1}$ segments of the curve can be chosen to correspond. Thus $\overline{\gamma_{3}}$ is generated by loops of the form $\alpha * \beta * \alpha^{-1}$ lying in $B\left(p_{2}, R_{2}\right)$, where $\beta$ is a closed path lying in a ball of radius $\delta_{2}$ and $\alpha$ is a path from $p_{2}$ to $\beta(0)$. So it is trivial.

Last, we need to show that $\Phi$ is onto. If $\bar{\alpha} \in G\left(p_{2}, r_{2}, R_{2}, \delta_{2}\right)$, it can be represented by some rectifiable closed curve $\sigma$ in $B\left(p_{2}, r_{2}\right)$ based at $p_{2}$. Choose an $\epsilon$-partition $\left\{y_{0}, \cdots, y_{m}\right\}$ of $\sigma$. Since $f: B\left(p_{1}, r_{1}\right) \rightarrow B\left(p_{2}, r_{2}\right)$ is an $\epsilon$-Hausdorff approximation, there are $x_{i} \in B\left(p_{1}, r_{1}\right), y_{i}^{\prime}=f\left(x_{i}\right) \in B\left(p_{2}, r_{2}\right)$, where $y_{0}^{\prime}=y_{m}^{\prime}=p_{2}$, $x_{0}=x_{m}=p_{1}$ and $d_{B\left(p_{2}, r_{2}\right)}\left(y_{i}, y_{i}^{\prime}\right) \leq \epsilon$. Connect $y_{i}^{\prime}, y_{i+1}^{\prime}$ with a length minimizing curve in $B\left(p_{2}, r_{2}\right)$; this yields a piecewise length minimizing closed curve $\sigma^{\prime}$ in $B\left(p_{2}, r_{2}\right)$ based at $p_{2}$, each segment having length $\leq 3 \epsilon$. So $\left[\sigma^{\prime}\right]=[\sigma]$ in $G\left(p_{2}, r_{2}, R_{2}, \delta_{2}\right)$. Now connect $x_{i}, x_{i+1}$ by length minimizing curves in $B\left(p_{1}, r_{1}\right)$. This yields a piecewise length minimizing $\gamma:[0,1] \rightarrow B\left(p_{1}, r_{1}\right)$ with base point $p_{1}$, each segment having length $\leq 4 \epsilon$. So the curve $\gamma$ allows a $5 \epsilon$-partition and $[\gamma] \in G\left(p_{1}, r_{1}, R_{1}, \delta_{1}\right)$. By the construction, $\Phi([\gamma])=\bar{\alpha}$.

Therefore $\Phi$ is surjective.

We prove a local relative version of Theorem 3.6 in SoWei]. Namely the GromovHausdorff limit of relative delta covers is close to being a relative delta cover of the limit.

Theorem 2.9. Suppose that $\left(M_{i}, p_{i}\right)$ converges to $(Y, y)$ in the pointed GromovHausdorff topology. Fix any $R>3 r>0$, and let $r_{i}, R_{i}$ be chosen such that $B\left(p_{i}, r_{i}\right)$ $\rightarrow B(y, r)$ and $B\left(p_{i}, R_{i}\right) \rightarrow B(y, R)$ with intrinisic metrics. If $\left(\tilde{B}\left(p_{i}, r_{i}, R_{i}\right)^{\delta}\right.$, $\left.d_{\tilde{B}\left(p_{i}, r_{i}, R_{i}\right)^{\delta}}, \tilde{p}_{i}\right)$ converges in the pointed Gromov-Hausdorff metric to some space which we will denote $\left(B(p, r, R)^{\delta}, d_{B(p, r, R)^{\delta}}, \tilde{p}\right)$, then $B(p, r, R)^{\delta}$ is a covering space of $B(p, r)$. Furthermore, for all $\delta_{2}>\delta>\delta_{1}, \tilde{B}(p, r, R)^{\delta_{1}}$ covers $B(p, r, R)^{\delta}$ and $B(p, r, R)^{\delta}$ covers $\tilde{B}(p, r, R)^{\delta_{2}}$. Namely we have covering projections

$$
\tilde{B}(p, r, R)^{\delta_{1}} \rightarrow B(p, r, R)^{\delta} \rightarrow \tilde{B}(p, r, R)^{\delta_{2}} \rightarrow B(p, r) .
$$

Proof. By Corollary [5.2, there exist sequences $r_{i} \rightarrow r, R_{i} \rightarrow R$, and maps $f_{i}$ such that for all $\delta>0$ there exists $N_{\delta}(r, R)$ such that for all $i \geq N_{\delta}(r, R)$ the 
maps $f_{i}: B(y, R) \rightarrow B\left(p_{i}, R_{i}\right)$ are $\delta$-Hausdorff approximations with respect to the intrinsic distances, and their restrictions $f_{i}: B(y, r) \rightarrow B\left(p_{i}, r_{i}\right)$ are also $\delta$ Hausdorff approximations with respect to the intrinsic distances on these smaller balls. So $B\left(p_{i}, r_{i}\right)$ and $B\left(p_{i}, R_{i}\right)$ converge to $B(p, r)$ and $B(p, R)$ with respect to intrinsic metrics.

Let $\pi^{\delta}\left(r_{i}, R_{i}\right):\left(\tilde{B}\left(p_{i}, r_{i}, R_{i}\right)^{\delta}, d_{\tilde{B}\left(p_{i}, r_{i}, R_{i}\right)^{\delta}}\right) \rightarrow\left(B\left(p_{i}, r_{i}\right), d_{B\left(p_{i}, r_{i}\right)}\right)$ be the covering map. It is distance nonincreasing by construction. After possibly passing to a subsequence it follows from a generalized version of the Arzela-Ascoli theorem (see GP, also [Pe, Page 279, Lemma 1.8]) that $\pi^{\delta}\left(r_{i}, R_{i}\right)$ will converge to a distance nonincreasing map $\pi^{\delta}(r, R): B(p, r, R)^{\delta} \rightarrow B(p, r)$.

By Lemma 2.3 the covering map

$$
\pi^{\delta}\left(r_{i}, R_{i}\right):\left(\tilde{B}\left(p_{i}, r_{i}, R_{i}\right), d_{\tilde{B}\left(p_{i}, r_{i}, R_{i}\right)}\right) \rightarrow\left(B\left(p_{i}, r_{i}\right), d_{B\left(p_{i}, r_{i}\right)}\right)
$$

is an isometry on balls of radius $\delta / 3$. So the limit projection $\pi^{\delta}(r, R): B(p, r, R)^{\delta} \rightarrow$ $B(p, r)$ is also an isometry on balls of radius $\delta / 3$ and $B(p, r, R)^{\delta}$ is a covering space of $B(p, r)$.

By the Unique Lifting Theorem [Ma, Lemma 3.1, Page 123] if $\tilde{Y}_{1}$ and $\tilde{Y}_{2}$ are covers of $Y$, then $\tilde{Y}_{1}$ covers $\tilde{Y}_{2}$ if every closed curve in $Y$ which lifts to a closed curve in $\tilde{Y}_{1}$ also lifts to a closed curve in $\tilde{Y}_{2}$.

Since $\pi^{\delta}(r, R)$ is an isometry on balls of radius $\delta / 3$ we have the covering projections

$$
\pi: \tilde{B}(p, r)^{\delta / 3} \rightarrow B(p, r, R)^{\delta} \rightarrow B(p, r) .
$$

To show the first projection in (2.9), it is enough to show that for $\delta / 3<\delta_{1}<\delta$, we have that $\tilde{B}(p, r, R)^{\delta_{1}}$ covers $B(p, r, R)^{\delta}$. We will use (2.11) to view $\tilde{B}(y, r, R)^{\delta_{1}}$ from above.

We can look at $\tilde{B}(p, r, R)^{\delta_{1}}$ as $\tilde{B}(p, r)^{\delta / 3} / \sim$, where $a \sim b$ iff there is a curve $\tilde{C}$ from $a$ to $b$ which projects to $C$ in $B(p, r)$ that is homotopic to a combination of $\alpha \circ \beta \circ \alpha^{-1}$ in $B(p, R)$ where $\beta \subset B_{q}\left(\delta_{1}\right)$.

From Theorem 2.7, there are homomorphisms $\phi_{i}$ from $G\left(p, r, R, \delta_{1}\right)$ to $G\left(p_{i}, r_{i}, R_{i}, \delta\right)$ for all $i$ large. Let $C_{i}=\phi_{i}([C]) \in B\left(p_{i}, r_{i}\right)$. Then $C_{i}$ converges to $C$ and they lift as closed curves $\tilde{C}_{i}$ to $\tilde{B}\left(p_{i}, r_{i}, R_{i}\right)^{\delta}$. Then we can look at the limit $\tilde{C}_{\infty} \in B(p, r, R)^{\delta}$ which is also a closed curve and is in fact the lift of $C$ in $B(p, r, R)^{\delta}$. Thus $\pi(a)=\pi(b)$, where $\pi$ is the covering map defined in (2.11). This allows us to define a map $\pi_{*}$ from equivalence classes of points in $\tilde{B}(p, r)^{\delta / 3}$ to $B(p, r, R)^{\delta}$. That is, $\pi_{*}: \tilde{B}(p, r, R)^{\delta_{1}} \rightarrow B(p, r, R)^{\delta}$.

Since $\pi=\pi_{2} \cdot \pi_{*}$, where $\pi_{2}$ is the natual covering projection from $\tilde{B}(p, r)^{\delta / 3}$ to $\tilde{B}(p, r, R)^{\delta_{1}}$, and $\pi$ is a covering projection, we can show $\pi_{*}$ commutes with natural covering projections $B(p, r, R)^{\delta}$ to $B(p, r)$ and $\tilde{B}(p, r, R)^{\delta_{1}}$ to $B(p, r)$. Therefore $\pi_{*}$ is a covering map by [Ma, Page 131, Lemma 6.7].

We now prove the other part of covering maps in (2.9):

$$
B(p, r, R)^{\delta} \rightarrow \tilde{B}(p, r, R)^{\delta_{2}} \rightarrow B(p, r) \forall \delta_{2}>\delta .
$$

Suppose there is a $\delta_{2}$ which is not a covering map. Then there is a closed curve $C$ in $B(p, r)$ whose lift to $B(p, r, R)^{\delta}$ is closed but whose lift to $\tilde{B}(p, r, R)^{\delta_{2}}$ is not a closed loop.

Since the lift of $C$ in $\tilde{B}(p, r, R)^{\delta_{2}}$ is not closed, $\Phi_{\delta_{2}}([C]) \in G\left(p, r, R, \delta_{2}\right)$ is nontrivial. Using Corollary [2.8 we can find $N$ sufficiently large so that $\Phi_{i}$ : 
$G\left(p_{i}, r_{i}, R_{i}, \delta\right) \rightarrow G\left(p, r, R, \delta_{2}\right)$ is surjective. In particular we can find curves $C_{i}$ which converge to $C$ in the Gromov-Hausdorff sense, such that $\Phi_{i}\left(\left[C_{i}\right]\right)=[C]$. Since $\left[C_{i}\right]$ are nontrivial, their lifts to $\tilde{B}\left(p_{i}, r_{i}, R_{i}\right)^{\delta}$ run between points $\tilde{C}_{i}(0) \neq \tilde{C}_{i}(1)$.

Furthermore, by Lemma 2.6

$$
d_{\tilde{B}\left(p_{i}, r_{i}, R_{i}\right)^{\delta}}\left(\tilde{C}_{i}(0), \tilde{C}_{i}(1)\right)=d_{\tilde{B}\left(p_{i}, r_{i}, R_{i}\right)^{\delta}}\left(\tilde{C}_{i}(0),\left[C_{i}\right] \tilde{C}_{i}(0)\right) \geq l\left(\left[C_{i}\right], r_{i}, R_{i}, \delta\right) \geq \delta .
$$

In the limit, the lifted curves $\tilde{C}_{i}$ converge to the lift of the limit of the curves, $\tilde{C}$ in $B(p, r, R)^{\delta}$ and

$$
d_{B(p, r, R)^{\delta}}(\tilde{C}(0), \tilde{C}(1)) \geq \delta .
$$

This implies that $\tilde{C}$ is not closed and we have a contradiction.

\section{Relative $\delta$-COvers With Ric $\geq(n-1) H$}

3.1. Gromov's precompactness extended. In order to apply Theorem 2.9. we need to prove that sequences of relative delta covers have Gromov-Hausdorff limits even though they are manifolds with boundary (Proposition 3.1). We show this can be done in the case when the balls are in manifolds with lower bounds on Ricci curvature. Recall that Gromov's Precompactness Theorem states that if $M_{i}^{n}$ are a sequence of complete $n$-dimensional manifolds with Ric $\geq(n-1) H$, then a subsequence of $M_{i}^{n}$ converge to a complete length space $Y$ Gr. There are two equivalent ways to describe this convergence of noncompact spaces as can be seen in Definition 5.1, Definition 5.2 and Lemma 5.1 of the Appendix.

We now state our precompactness result.

Proposition 3.1. Let $M_{i}^{n}$ be complete $n$-dimensional Riemannian manifolds with $R i c \geq(n-1) H$ that converges to a length space $Y$. Fix any $R>0$ and $r \in(0, R / 3)$. Let $r_{i}$ and $R_{i}$ be radii such that $B\left(p_{i}, r_{i}\right)$ and $B\left(p_{i}, R_{i}\right)$ in $M_{i}$ with intrinsic metrics converge to $B(y, r)$ and $B(y, R)$ in $Y$ with intrinsic metrics. Then for all $\delta \in$ $(0, r / 2)$ the relative $\delta$ covers, $\left(\tilde{B}_{i}\left(p_{i}, r_{i}, R_{i}\right)^{\delta}, \tilde{p}_{i}\right)$, with intrinsic metric $d_{\tilde{B}_{i}\left(p_{i}, r_{i}, R_{i}\right)^{\delta}}$, have a convergent subsequence in the pointed Gromov-Hausdorff sense.

Note that the universal cover of the balls themselves need not have a converging subsequence in the pointed Gromov-Hausdorff sense even when they are given a nonnegative sectional curvature condition (Example 3.2).

To prove Proposition 3.1 we need several lemmas. By Gromov's Precompactness Theorem [Pe, Page 280, Lemma 1.9], Gr], it is enough to bound the number of $\epsilon$-net points, the centers of the minimal set of $\epsilon$-balls whose union covers a ball of a given radius centered at $\tilde{p}_{i}$ in $\tilde{B}_{i}\left(p_{i}, r_{i}, R_{i}\right)^{\delta}$, uniformly for all $i$. This can be done by bounding the maximum number of disjoint balls of $\epsilon / 2$ in any ball of fixed radius $B_{\tilde{p}_{i}}(s)$.

Using the Bishop-Gromov volume comparison theorem, the $\epsilon$-net points can be easily bounded in arbitrarily large balls within complete spaces with a lower bound on Ricci curvature. However, $\widetilde{B}(p, r, R)^{\delta}$ is not a complete manifold and neither is $\tilde{B}(p, R)^{\delta}$. Note that $\tilde{B}(p, R)^{\delta}$ could have exponential, not polynomial, volume growth (see Example 1.3). Nevertheless, we can prove that Bishop-Gromov volume comparison does hold for small balls in $\tilde{B}(p, R)^{\delta}$ and then use other techniques to count larger nets. 
We begin with some illustrative examples.

Example 3.2. Let $Y$ be a singular $\mathbb{R} P^{2}$, viewed as gluing the opposite points of the boundary of a flat disk with center $y$ and radius 1 .

Now let $X_{k}$ be similar copies of singular $\mathbb{R} P^{2}$ by gluing the opposite points of a regular $2 k$-polygon inscribed in the unit disk. Let $x_{k}$ be the center points and $r_{k}=\frac{\cos \frac{\pi}{2 k}+3}{4}$ so that $B\left(x_{k}, r_{k}\right)$ reaches the boundary of the $2 k$ polygon but is within the unit disk. Then the fundamental groups of $B\left(x_{k}, r_{k}\right)$ in $X_{k}$ have a free group with $k$ generators.

Clearly $\left(X_{k}, x_{k}\right)$ converge to $(Y, y)$ in Gromov-Hausdorff sense but the universal covers of $B\left(x_{k}, r_{k}\right), \tilde{B}\left(x_{k}, r_{k}\right)$ do not converge in the Gromov-Hausdorff sense, nor do they have any converging subsequence. This can be seen because the number of disjoint balls of radius $\cos (\pi /(2 k))$ in $B_{\tilde{x}_{k}}(2) \subset \tilde{B}\left(x_{k}, r_{k}\right)$ is at least $2 k+1$ and diverges to infinity.

Now $X_{k}$ can be smoothed to manifolds $M_{k}$ which are diffeomorphic to $\mathbb{R} P^{2}$ and have nonnegative sectional curvature. To construct $M_{k}$, first smooth the $X_{k}$ at the corner of the polygons, then curve down the boundary so that the boundary is totally geodesic and a reflection of this piece across the boundary is a smooth $S^{2}$. Let $M_{k}$ be this sphere's $\mathbb{Z}_{2}$ quotient. If we do the smoothing so that region of smoothing is very small, then $M_{k}$ also converges to $Y$ and the fundamental group of $B\left(p_{k}, r_{k}^{\prime}\right)$ in $M_{k}$ with $r_{k}^{\prime}=\frac{3 \cos \frac{\pi}{2 k}+1}{4}$ also has a free group with $\mathrm{k}$ generators. By rescaling the manifolds $M_{k}$ we have a sequence of smooth 2-dimensional manifolds $M_{k}$ with nonnegative sectional curvature such that the universal cover of the balls of radius 1 in $M_{k}$ have no converging subsequence.

The following example shows the difference between a delta cover and a relative delta cover, illustrating why balls are controlled better in the latter case.

Example 3.3. Let $M=T^{4}$ such that the first two circles have diameter 1 and the second two have diameter 5 as length spaces. Let $R=8$ and $r=2$. Note that $\tilde{B}(p, R)$ and $\tilde{B}(p, r)$ both have exponential volume growth since $\tilde{B}(p, R)=\mathbb{R}^{2} \times X$, where $X$ branches in two dimensions causing exponential growth on top of the polynomial growth of order 2 , and $\tilde{B}(p, r)$ also has the branching effect in the first two dimensions but does not have a Euclidean factor. However the relative $\delta$ cover is under control because the nontrivial directions of the connected lift of $B(p, r)$ to $\tilde{B}(p, R)$ are lifted into the Euclidean factor and thus cannot grow more than polynomially.

In the following lemmas we will omit the subscript $i$ and just proceed to find a uniform bound on the number of minimal sets of $\epsilon$-balls whose union covers a ball of fixed radius centered at $\tilde{p}$ in $\tilde{B}(p, r, R)^{\delta}$ depending only on $n, r, R$ and $H$.

We first have a lemma concerning bounded balls measured using the restricted metric.

Lemma 3.4. Let $M^{n}$ be a manifold with Ric $\geq(n-1) H$. If $B(\tilde{p}, s) \subset \tilde{B}(p, R)^{\delta}$ is centered on a lift of $p$ and is measured with the metric restricted from $d_{\tilde{B}(p, R)^{\delta}}$, then we have

$$
\frac{\operatorname{vol}\left(B\left(\tilde{p}, s_{1}\right)\right)}{\operatorname{vol}\left(B\left(\tilde{p}, s_{2}\right)\right)} \geq V\left(n, H, s_{1}\right) / V\left(n, H, s_{2}\right) \quad \forall s_{1}<s_{2} \leq R .
$$


Also for all $\tilde{x} \in \tilde{B}(p, R)^{\delta}$, we have

$$
\frac{\operatorname{vol}\left(B\left(\tilde{x}, s_{1}\right)\right)}{\operatorname{vol}\left(B\left(\tilde{x}, s_{2}\right)\right)} \geq V\left(n, H, s_{1}\right) / V\left(n, H, s_{2}\right) \quad \forall s_{1}<s_{2} \leq R-h,
$$

where $h$ is the distance from $\tilde{x}$ to the nearest lift of $p$, and $V(n, H, r)$ is the volume of balls of radius $r$ in the model space $M_{H}^{n}$, the $n$-dimensional simply connected Riemannian manifold with constant sectional curvature $H$.

Proof. Note that the proof of Bishop-Gromov volume comparison [BiCr, $[\mathrm{Gr}]$ can easily be applied here as long as we have smooth minimal geodesics, so we need only avoid the boundary of $\tilde{B}(p, R)^{\delta}$. That is, we can apply it to balls $B(\tilde{x}, s)$ such that all points $\tilde{q}$ in the ball are joined to $\tilde{x}$ by a minimal geodesic that avoids the boundary of $\tilde{B}(p, R)^{\delta}$. This works for $\tilde{x}=\tilde{p}$ as long as $s$ is less than $R$, since there will be a curve of length $s$ joining $\tilde{p}$ to $\tilde{q}$ and this curve will project to a curve in $B(p, R)$ starting from $p$. If the curve hits the boundary, then its projection hits the boundary of $B(p, R)$, so it has length $\geq R$.

For arbitrary $\tilde{x}$, we avoid the boundary by staying inside a ball $B(g \tilde{p}, R)$ for the lift of $p$ closest to $\tilde{x}$.

This has a corollary which gives a volume estimate for bounded balls on $\tilde{B}(p, r, R)^{\delta}$ with its intrinsic length metric. We state four versions of the estimates because they will be needed later to construct a measure on the limit space.

Corollary 3.5. Let $B(\tilde{x}, s) \subset \tilde{B}(p, r, R)^{\delta}$ be a ball of radius $s$ measured using $d_{\tilde{B}(p, r, R)^{\delta}}$.

Then if $\tilde{x}=\tilde{p}, s_{1}<r$ and $s_{1}<s_{2}<R$ we have

$$
\frac{\operatorname{vol}\left(B\left(\tilde{p}, s_{1}\right)\right)}{\operatorname{vol}\left(B\left(\tilde{p}, s_{2}\right)\right)} \geq V\left(n, H, s_{1}\right) / V\left(n, H, s_{2}\right) .
$$

Furthermore for $\tilde{x}_{1}, \tilde{x}_{2} \in \tilde{B}(p, r, R)^{\delta}$ with $s=d_{\tilde{B}(p, r, R)^{\delta}}\left(\tilde{x}_{1}, \tilde{x}_{2}\right)$ we get the following inequalities:

(3.4)

$$
\frac{\operatorname{vol}\left(B\left(\tilde{x}_{1}, r_{1}\right)\right)}{\operatorname{vol}\left(B\left(\tilde{x}_{2}, r_{2}\right)\right)} \geq \frac{V\left(n, H, r_{1}\right)}{V\left(n, H, r_{2}+s\right)} \quad \forall r_{1} \leq r_{2} \leq R-r-s, r_{1}<r-d_{M}\left(\pi\left(\tilde{x}_{1}\right), p\right),
$$

$$
\begin{aligned}
& \frac{\operatorname{vol}\left(B\left(\tilde{x}_{2}, r_{2}\right)\right)}{\operatorname{vol}\left(B\left(\tilde{x}_{1}, r_{1}\right)\right)} \geq \frac{V\left(n, H, r_{2}\right)}{V\left(n, H, r_{1}+s\right)} \quad \forall r_{2} \leq r_{1}+s \leq R-r, r_{2}<r-d_{M}\left(\pi\left(\tilde{x}_{2}\right), p\right), \\
& \frac{\operatorname{vol}\left(B\left(\tilde{x}_{2}, r_{2}\right)\right)}{\operatorname{vol}\left(B\left(\tilde{x}_{1}, r_{1}\right)\right)} \geq 1 \quad \forall r_{2} \geq r_{1}+s .
\end{aligned}
$$

Proof. Note that if $B(\tilde{p}, s)$ is measured with $d_{\tilde{B}(p, r, R)^{\delta}}$, then it is a subset of $B(\tilde{p}, s)$ with $d_{\tilde{B}(p, R)^{\delta}}$. For $s_{1}<r-d_{M}(\pi(\tilde{x}), p), B\left(\tilde{p}, s_{1}\right)$ is the same whether it is measured with $d_{\tilde{B}(p, r, R)^{\delta}}$ or with $d_{\tilde{B}(p, R)^{\delta}}$ because it avoids the boundary. Thus (3.3) follows from Lemma 3.4 which only allows $s_{2} \leq R$.

We now prove the equations needed to create a measure on the limit spaces. Before applying (3.2), we note that $h$ does not depend on the metric which is used to measure it, intrinsic or restricted. Furthermore, here $h \leq r$.

We get (3.4) by setting $s_{1}=r_{1}<r, s_{2}=r_{2}+s$ and $\tilde{x}=\tilde{x}_{1}$. So we need $r_{2}+s \leq R-r$ and $r_{1}<r-d_{M}(\pi(\tilde{x}), p)$. 
We get (3.5) by setting $s_{1}=r_{2}, s_{2}=r_{1}+s$ and $\tilde{x}=\tilde{x}_{2}$. So we need $r_{1}+s \leq R-r$ and $r_{2}<r-d_{M}(\pi(\tilde{x}), p)$.

At this point we need to control larger regions than just balls of radius $<R$. We do not have a volume comparison theorem on this scale, but we can control these regions by piecing together fundamental domains and controlling the generators $g \in G(p, r, R, \delta)$ which map a fundamental domain based at $\tilde{p}$ to an adjacent fundamental domain based at $g \tilde{p}$.

Lemma 3.6. Let $M^{n}$ be a Riemannian manifold with Ric $\geq(n-1) H$. Let FD be the Dirichlet fundamental domain of $B(p, r)$ based at $\tilde{p} \in \tilde{\tilde{B}}(p, r, R)^{\delta}$ with $3 r<R$ (see Definition 2.4).

Then the number of $g F D \subset B(\tilde{p}, 3 r)$ with the ball measured using the restricted metric, $d_{\tilde{B}(p, r, R)^{\delta}}$, is uniformly bounded by a number $N=N(n, H, r, \delta)$. So is the number of $g F D$ of the connected lift which are adjacent to $F D$ (i.e. $g F D \cap F D \neq \emptyset$ ).

Proof. Note that if $g F D$ is adjacent to $F D$, then $g F D \subset B(\tilde{p}, 3 r)$ with the ball measured using the restricted metric, $d_{\tilde{B}(p, r, R)^{\delta}}$. Furthermore $\delta$ balls around $\tilde{p}$ and $g \tilde{p}$ are isometric and disjoint. So we can apply the volume comparison above (Lemma 3.4) and packing arguments to get that

$$
N \leq \operatorname{vol}(B(\tilde{p}, 3 r)) / \operatorname{vol}(B(\tilde{p}, \delta)) \leq V(n, H, 3 r) / V(n, H, \delta) .
$$

We also need the following net lifting lemma which does not require a Ricci curvature bound.

Lemma 3.7. If $\mathcal{A}$ is an $\epsilon$-net point of $B(p, r-\epsilon)$ (we can assume that $\mathcal{A} \subset$ $B(p, r-\epsilon))$ with restricted metric from $d_{M}$ and $\epsilon<\delta$, then the lift of $\mathcal{A}$ is a $2 \epsilon-$ net point of $\tilde{B}(p, r, R)^{\delta}(3 r<R)$ and its fundamental domains with respect to the intrinsic metric $d_{\tilde{B}(p, r, R)^{\delta}}$. Furthermore the number of $2 \epsilon$-balls needed to cover each gFD is less than or equal to $N \operatorname{card}(\mathcal{A})$, where $N$ is defined in Lemma 3.6 .

Proof. Since $\epsilon<\delta$, the covering map $\pi: \tilde{B}(p, r, R)^{\delta} \rightarrow B(p, r)$ is a diffeomorphism from $U_{\tilde{q}}$ to $B_{q}(\epsilon), d_{M}$, where $U_{\tilde{q}}$ is the connected component of a lift of $B_{q}(\epsilon)$ centered at a lift $\tilde{q}$ of $q$.

Note that $U_{\tilde{q}}$ is not necessarily isometric to nor contained in a ball of radius $\epsilon$ measured using $d_{\tilde{B}(p, r, R)^{\delta}}$. However, it is easy to see that

$$
F D \cap \pi^{-1}(B(p, r-\epsilon)) \subset \bigcup_{q \in \mathcal{A}} \bigcup_{g \text { s.t.gFD } D \cap D \neq \emptyset} U_{g \tilde{q}}
$$

which is a union of $N \operatorname{card}(\mathcal{A})$ sets. Here each $\tilde{q}$ is a chosen lift of $q$ to $F D$.

For any $\tilde{y} \in F D \subset \tilde{B}(p, r, R)^{\delta}$, there is $y \in B(p, r)$ which is joined by a minimal geodesic to $p$. Thus there is a point $x \in B(p, r-\epsilon)$ joined to $y$ by a smooth geodesic of length $\leq \epsilon$. This lifts upstairs and we get a point $\tilde{x} \in F D \cap \pi^{-1}(B(p, r-\epsilon))$ joined to $\tilde{y}$ by a smooth geodesic contained in $\tilde{B}(p, r, R)^{\delta}$ of length $\leq \epsilon$.

For any $\tilde{x} \in F D \cap \pi^{-1}(B(p, r-\epsilon))$, by (3.8), there exists a $q \in \mathcal{A}$ and a $g$ such that $\tilde{x} \in U_{g \tilde{q}}$. So $\tilde{x}$ projects to a point $x$ in $B_{q}(\epsilon) \subset B(p, r)$ measured using $d_{M}$. Thus $x$ has a smooth minimal geodesic contained in $B(p, r)$ of length $<\epsilon$ joining it to $q$, and this lifts to a smooth curve in $\tilde{B}(p, r, R)^{\delta}$ joining $\tilde{x}$ to $g \tilde{q}$. In conclusion, 
for all $\tilde{y} \in F D$ we have a curve of length $<2 \epsilon$ contained in $\tilde{B}(p, r, R)^{\delta}$ joining $\tilde{y}$ to a $g \tilde{q}$ :

$$
F D \subset \bigcup_{q \in \mathcal{A}} \bigcup_{g \text { s.t.gF } D \cap F D \neq \emptyset} B_{g \tilde{q}}(2 \epsilon),
$$

where the balls in the union are measured using the intrinsic metric $d_{\tilde{B}(p, r, R)^{\delta}}$.

Now we are ready to prove Proposition 3.1 .

Proof of Proposition 3.1. Let $B(\tilde{p}, \bar{R})$ be any large ball in $\left(\tilde{B}(p, r, R)^{\delta}, d_{\tilde{B}(p, r, R)^{\delta}}\right)$. Note that any $\epsilon / 2$-net of $B(p, r-\epsilon)$ with restricted metric from $d_{M}$ is uniformly bounded by volume comparison $[\mathrm{Gr}$. Hence we just need to bound the number of fundamental domains intersecting $B(\tilde{p}, \bar{R})$ so we can lift the $\epsilon / 2$-net from $B(p, r-\epsilon / 2)$ and give a bound on $\epsilon$-net points of $B(\tilde{p}, \bar{R})$ using Lemma 3.7 .

Suppose FD is a fundamental domain of $B(p, r)$ based at $\tilde{p}$ and $g \mathrm{FD}$ intersects $B(\tilde{p}, \bar{R})$. Then there is a curve of length $\leq \bar{R}$ running from a point in $g$ FD back to $\tilde{p}$ which stays in $\tilde{B}(p, r, R)^{\delta}$. This curve is contained in a union of $k$ fundamental domains each of which is adjacent to the next. These fundamental domains can be called $h_{j} F D$ with $h_{0}=e$ and $h_{k}=g$. So we can write $g=g_{1} g_{2} \ldots g_{k}$, where $g_{1}=h_{1}, g_{2}=h_{1}^{-1} h_{2}, \ldots, g_{k}=h_{k-1}^{-1} h_{k}$. Note that each $g_{j}$ is a generator (that is, $g_{j} \mathrm{FD}$ is adjacent to FD). So if we can find a uniform bound on $k$, then the number of fundamental domains $g$ FD intersecting the ball of radius $\bar{R}$ is bounded by the number of words of length $k$ in $N$ generators, which is $N^{k}$.

By Lemma [3.6] $N \leq N(n, H, r, \delta)$. We also get that $\bar{N}$, the number of deck transforms $g$ such that $g \tilde{p}$ is in $\tilde{B}(p, r, R)^{\delta}$ and $d(g \tilde{p}, \tilde{p})<3 r$, is $\leq N(n, H, r, \delta)$.

We will show that $k<\bar{N}([\bar{R} / r]+1)$, where $[\bar{R} / r]$ is the integer part of $\bar{R} / r$. If not, then look at a sequence of points $q_{0}=\tilde{p}, \ldots, q_{j} \in h_{j} F D, \ldots$ all running along the curve of length $\leq \bar{R}$. The last point is $q_{k}$. If $k \geq \bar{N}([\bar{R} / r]+1)$, then we can look at the points $q_{0}, q_{\bar{N}}, q_{2 \bar{N}}, q_{([\bar{R} / r]+1) \bar{N}}$. Each pair of these points has $\bar{N}-1$ points lying between them. Furthermore $\min d\left(q_{j \bar{N}}, q_{(j+1) \bar{N}}\right) \leq \bar{R} /([\bar{R} / r]+1)<r$, otherwise $d\left(q_{0}, q_{k}\right)>\bar{R}$. Thus there exist $\bar{N}+1$ points which are within a distance $r$ from the minimizing $q_{j \bar{N}}$. (That is, all the points up to and including $q_{(j+1) \bar{N}}$.)

Now each of these $q_{k} \in h_{k} F D$, so $d\left(h_{k} \tilde{p}, q_{k}\right)<r$. Thus we know there exist $\bar{N}+1$ points of the form $g \tilde{p}$ which are within a distance $3 r$ from $h_{j \bar{N}} \tilde{p}$ in the length metric and thus also in the restricted metric. Multiplying all points by $h_{j \bar{N}}^{-1}$ we contradict the definition of $\bar{N}$. Thus the claim which bounds $k$ is correct, and we are done.

3.2. Renormalized measures. By the previous two sections, we know that if $M_{i}^{n}$ are complete manifolds with Ric $\geq(n-1) H$, then for any $p_{i} \in M_{i}$ and any $R>3 r>0$, there is a subsequence of the $i$ such that $\left(M_{i}, p_{i}\right)$ converge to $(Y, y)$ and there exist $r_{i} \rightarrow r$ and $R_{i} \rightarrow R$ such that $\left(B\left(p_{i}, r_{i}\right), p_{i}\right) \rightarrow(B(y, r), y)$ and the relative delta covers $\tilde{B}\left(p_{i}, r_{i}, R_{i}\right)^{\delta} \rightarrow B(y, r, R)^{\delta}$, where $B(y, r, R)^{\delta}$ covers $B(y, r)$ for any fixed $\delta>0$. We now construct a renormalized limit measure on $B(y, r, R)^{\delta}$ similar to the one used by Cheeger and Colding to construct a limit measure on $Y$. In fact we prove the following more general theorem which allows us to vary the $\delta$ in the sequence of relative delta covers.

Proposition 3.8. Let $M_{i}^{n}$ be complete manifolds with Ric $\geq(n-1) H$ such that $G H \lim _{i \rightarrow \infty}\left(M_{i}, p_{i}\right)=(Y, y)$. Fix $R \in(0, \infty]$. Then for all $r \in(0, R / 4)$ and 
$\delta_{i} \in(0, r / 2)$ and $r_{i} \rightarrow r$ such that

$$
\left(B(y, r), d_{B(y, r)}\right)=G H \lim _{i \rightarrow \infty}\left(B\left(p_{i}, r_{i}\right), d_{B\left(p_{i}, r_{i}\right)}\right),
$$

the relative $\delta_{i}$ covers converge to a covering space $\hat{B}(y, r, R)$ for some $R_{i} \rightarrow R \in$ $(0, \infty]$ as follows:

$$
\left(\hat{B}(y, r, R), \hat{y}, d_{\hat{B}(y, r, R)}\right):=G H \lim _{i \rightarrow \infty}\left(\tilde{B}_{i}\left(p_{i}, r_{i}, R_{i}\right)^{\delta_{i}}, \tilde{p}_{i}, d_{\tilde{B}_{i}\left(p_{i}, r_{i}, R_{i}\right)^{\delta_{i}}}\right),
$$

and $\pi: \hat{B}(y, r, R) \rightarrow B(y, r)$ is a limit of covering maps $\pi_{i}: \tilde{B}\left(p_{i}, r_{i}, R_{i}\right)^{\delta_{i}} \rightarrow$ $B\left(p_{i}, r_{i}\right)$ all of which are isometries on balls of some common radius $\delta / 2>0$. Then $\hat{B}(y, r, R)$ has a renormalized limit measure, $\mu$, which is Borel regular. This measure is a limit of measures on a subsequence $i_{j}$ of the original sequence in the following sense: for all $\hat{x} \in \hat{B}(y, r, R), s<r-d_{Y}(\pi(\hat{x}), y)$ there exists $\tilde{x}_{i_{j}} \in \tilde{B}\left(p_{i_{j}}, r_{i_{j}}, R_{i_{j}}\right)^{\delta_{i_{j}}}$ such that

$$
\mu(B(\hat{x}, s))=\bar{V}_{\infty}(B(\hat{x}, s))=\lim _{i_{j} \rightarrow \infty} \frac{\operatorname{Vol}\left(B\left(\tilde{x}_{i_{j}}, s\right)\right)}{\operatorname{Vol}\left(B\left(\tilde{p}_{i_{j}}, r / 10\right)\right)} .
$$

In fact $\mu$ is created from its measure on these small balls using Caratheodory's Construction.

Furthermore, we have the Bishop-Gromov Volume Comparison,

$\frac{\mu\left(B\left(\hat{x}, r_{1}\right)\right)}{\mu\left(B\left(\hat{x}, r_{2}\right)\right)} \geq V\left(n, H, r_{1}\right) / V\left(n, H, r_{2}\right) \forall r_{1} \leq r_{2}<(R-r) / 2, \quad r_{1}<r-d_{Y}(\pi(\hat{x}), y)$.

Finally $\mu$ is Radon when restricted to closed sets contained in balls about $\hat{y}$ that avoid the boundary.

Note that we must assume that the limit of the relative delta covers exists and is a cover for this result, as can be seen in Example 3.2 Note also that when $\delta_{i}=\delta$ then $\hat{B}(y, r, R)=B(y, r, R)^{\delta}$ of Theorem 2.9 and all the conditions of Proposition 3.8 are satisfied. However, we do not in general assume that $\delta_{i}$ are bounded below by some $\delta$, just that all $\pi_{i}$ are isometries on $\delta / 2$ balls.

Recall that Caratheodory's Construction consists of taking a function $\psi: F \rightarrow \mathbb{R}$ where $F$ is a collection of sets and then taking an infimum as follows:

$$
\mu_{\epsilon}(A)=\inf \left\{\sum_{B \in G} \psi(B): G \subset F \cap\{B: \operatorname{diam}(B) \leq \epsilon\} \text { and } A \subset \bigcup_{B \in G} B\right\},
$$

and let

$$
\mu(A)=\lim _{\epsilon \rightarrow 0} \mu_{\epsilon}(A) .
$$

When all the members of $F$ are Borel sets, $\mu$ is a Borel Regular measure Fed. 2.10]. This measure is Radon when restricted to measurable sets by Theorem 13.7 of [Mun] which only requires that the members of $F$ are open sets.

Cheeger and Colding defined a function $\psi$ on all balls in $Y$ using the relative volume comparison theorem and taking limits of subsequences. They then quote a standard packing argument to create a uniform approximation of the infimum in (3.63). We do not have control over all balls, just those that avoid the boundary of $\tilde{B}\left(p_{i}, r_{i}, R_{i}\right)^{\delta_{i}}$. So in our case $F$ does not consist of all balls. Thus our measure only agrees with the one defined by Cheeger-Colding on sets whose tubular neighborhoods avoid the boundary as follows. 
Corollary 3.9. If the renormalized limit measure, $\mu_{Y}$, on $Y$ is defined as in ChCo2 with respect to balls of radius 1 and we take $\delta<\min \{r / 10,1\}$ in Proposition 3.8 then the measure $\mu_{\hat{B}(y, r, R)}$ on $\hat{B}(y, r, R)$ of Proposition 3.8 agrees up to a scale with $\mu_{Y}$ when evaluated on closed isometrically lifted sets $S$ contained in closed balls that avoid the boundary of $B(y, r)$. Namely

$$
\mu_{\hat{B}(y, r, R)}(S)=\lambda \mu_{Y}(\pi(S))
$$

where $\lambda=\lim _{i \rightarrow \infty} \frac{\operatorname{vol}\left(B\left(p_{i}, 1\right)\right)}{\operatorname{vol}\left(B\left(\tilde{p}_{i}, r / 10\right)\right)} \in\left[\frac{V(n, H, \delta / 2)}{V(n, H, r / 10)}, \frac{V(n, H, 1)}{V(n, H, \delta / 2)}\right]$.

This corollary will follow from the proof of Proposition 3.8 because the definition of $\bar{V}_{\infty}$ is exactly as in [ChCo2] and the Caratheodry constructions used in their paper and here will agree on sets which avoid the boundary. The upper bound for $\lambda$ is found using Bishop Gromov on $B\left(p_{i}, 1\right)$ and the fact that $B\left(p_{i}, \delta / 2\right)$ lifts isometrically and that $B\left(\tilde{p}_{i}, \delta / 2\right) \subset B\left(\tilde{p}_{i}, r / 10\right)$. The lower bound for $\lambda$ is found using Bishop Gromov on $B\left(\tilde{p}_{i}, r / 10\right)$ and the fact that $B\left(\tilde{p}_{i}, \delta / 2\right)$ is mapped isometrically to $B\left(p_{i}, \delta / 2\right) \subset B\left(p_{i}, 1\right)$.

The next corollary concerns both our measure and the one defined by CheegerColding. That is, $X_{i}$ can be taken to be complete manifolds converging to $X$, or $X_{i}$ can be $\tilde{B}\left(y_{i}, r_{i}, R_{i}\right)^{\delta_{i}}$ converging to $X=\hat{B}(y, r, R)$. In the former case $\operatorname{int}(X)=X$. The corollary will be proven at the end of this subsection.

Corollary 3.10. Suppose $X_{i}$ converges to $X$ with the renormalized measure convergence defined above. If $K_{i} \subset X_{i}$ converges to a compact set $K \in \operatorname{int}(X)$ as subsets of $X_{i}$ and $X$, then there exists a sequence $\epsilon_{i}>0$ converging to 0 such that

$$
\lim _{i \rightarrow \infty} \frac{\operatorname{vol}\left(T_{\epsilon_{i}}\left(K_{i}\right)\right)}{\operatorname{vol}\left(B\left(\tilde{p}_{i}, r / 10\right)\right)}=\mu(K)
$$

We need the following packing lemma before we can prove Proposition 3.8.

We first define special compact subsets of covers of $B(p, r)$ which avoid the boundary by a definite amount. Let $\pi: \tilde{B} \rightarrow B(p, r)$ be any cover and let $R_{0}<r$. Then we define

$$
K_{R_{0}}:=\left\{\tilde{x} \in \tilde{B}: d_{B(p, r)}(\pi(\tilde{x}), p) \leq R_{0}\right\}, \quad i \tilde{B}:=\bigcup_{R_{0}<r} K_{R_{0}} .
$$

Note that

$$
\pi(i \tilde{B})=B_{p}(r) \text { and } C l(i \tilde{B})=\tilde{B} .
$$

Lemma 3.11. Given $R>2 r, R_{0}<r, B(\tilde{x}, s) \subset \tilde{B}\left(p_{i}, r_{i}, R_{i}\right)^{\delta}$ (the subscript $i$ will be omitted below in this lemma) such that $2 s<R-r$. For any compact set $K \subset B(\tilde{x}, s) \cap K_{R_{0}}, \epsilon \in\left(0, \min \left\{r-R_{0}, r / 2\right\}\right)$ there exists $\lambda(\epsilon, s, n, H), N(\epsilon, s, n, H)$ and a collection of balls of radii, $r_{i} \in[\lambda, \epsilon]$ centered on $z_{i} \in K$ such that

$$
\bigcup_{i=1}^{N} B_{z_{i}}\left(r_{i}\right) \supset K \text { and } \sum_{i=1}^{N} \operatorname{vol}\left(B_{z_{i}}\left(r_{i}\right)\right) \leq(1+\epsilon) \operatorname{vol}(K),
$$

and there are $r_{i}^{\prime}<r_{i}$ such that $B_{z_{i}}\left(r_{i}^{\prime}\right)$ are disjoint subsets of $K$ such that

$$
\sum_{i=1}^{N} \operatorname{vol}\left(B_{z_{i}}\left(r_{i}^{\prime}\right)\right) \geq(1-\epsilon) \operatorname{vol}(K) \text {. }
$$


Proof. Fix $\beta<1$. Let $N_{0}$ be the maximum number of disjoint balls contained in $K_{0}=K$ of radius $\epsilon_{0}=\epsilon$. Let $S_{0}=\left\{z_{1}, \ldots, z_{N_{0}}\right\}$ be the centers of these balls and $r_{z}=\epsilon_{0}$ their radii.

Since $z_{i} \in K \subset K_{R_{0}}$, so $\epsilon<r-R_{0} \leq r-d_{M}\left(p, \pi\left(z_{i}\right)\right)$, thus we can apply (3.4) of Corollary 3.5 with an inner ball $B\left(z_{i}, \epsilon\right)$ and an outer ball up to radius $R-r$.

First we apply it to show that $N_{0}=N_{0}(\epsilon, s, n, H)$ does not depend on the manifold using the standard packing. Recall that $2 s<R-r$.

$$
\operatorname{vol}\left(B\left(z_{i}, \epsilon\right)\right) \geq \frac{V(n, H, \epsilon)}{V(n, H, 2 s)} \operatorname{vol}\left(B\left(z_{i}, 2 s\right)\right) \geq \frac{V(n, H, \epsilon)}{V(n, H, 2 s)} \operatorname{vol}(B(\tilde{x}, s)) .
$$

So

$$
\operatorname{vol}(B(\tilde{x}, s)) \geq \sum_{i=1}^{N_{0}} \operatorname{vol}\left(B\left(z_{i}, \epsilon\right)\right) \geq N_{0} \frac{V(n, H, \epsilon)}{V(n, H, 2 s-\epsilon)} \operatorname{Vol}(B(\tilde{x}, s)) .
$$

Second we apply (3.4) to estimate how much of the volume of $B(\tilde{x}, s)$ has been covered by these balls. Note that $2 r_{z}=2 \epsilon<r<R-r$.

$$
\begin{aligned}
\sum_{i=1}^{N_{0}} \operatorname{vol}\left(B_{z_{i}}\left(r_{z}\right)\right) & \geq \frac{V(H, n, \epsilon)}{V(H, n, 2 \epsilon)} \sum_{i=1}^{N_{0}} \operatorname{vol}\left(B_{z_{i}}\left(2 r_{z}\right)\right) \\
& \geq \frac{V(H, n, \epsilon)}{V(H, n, 2 \epsilon)} \operatorname{vol}(K) .
\end{aligned}
$$

Let

$$
K_{j}=K_{j-1} \backslash \bigcup_{z \in S_{j-1}} B_{z}\left(r_{z}\right)
$$

Let $N_{j}$ be the maximum number of disjoint balls contained in $K_{j}$ of radius $\epsilon_{j}=\beta^{j} \epsilon$. Let $S_{j}=\left\{z_{N_{0}+\cdots+N_{j-1}+1}, \ldots, z_{N_{0}+\cdots+N_{j}}\right\}$ be the centers of these balls and $r_{z}=\epsilon_{j}$ be their radii. As argued above, we can apply (3.4) with an inner ball $B\left(z_{i}, \epsilon_{j}\right)$, because these balls are contained in $B(\tilde{x}, s)$, and apply this to prove $N_{j}=N_{j}(\epsilon, \beta, s, n, H)$.

Now balls of twice the radius cover $K_{j}$ and we have

$$
\begin{aligned}
\sum_{i=N_{0}+\ldots+N_{j-1}+1}^{N_{0}+\ldots+N_{j}} \operatorname{vol}\left(B_{z_{i}}\left(r_{z}\right)\right) & \geq \frac{V\left(H, n, \beta^{j} \epsilon\right)}{V\left(H, n, 2 \beta^{j} \epsilon\right)} \sum_{i=N_{0}+\ldots+N_{j-1}+1}^{N_{0}+\ldots+N_{j}} \operatorname{vol}\left(B_{z_{i}}\left(2 r_{z}\right)\right) \\
& \geq \frac{V\left(H, n, \beta^{j} \epsilon\right)}{V\left(H, n, 2 \beta^{j} \epsilon\right)} \operatorname{vol}\left(K_{j}\right)=C(H, n, \beta, \epsilon, j) \operatorname{vol}\left(K_{j}\right),
\end{aligned}
$$

where

$$
C(H, n, \beta, \epsilon, j)=\frac{V\left(H, n, \beta^{j} \epsilon\right)}{V\left(H, n, 2 \beta^{j} \epsilon\right)} .
$$

For fixed $\epsilon, \beta<1$ we can take $J_{0}$ sufficiently large such that $C(H, n, \beta, \epsilon, J)$ is approximately $(1 / 2)^{n}$. More precisely we can take $J_{0}$ sufficiently large such that

$$
C(H, n, \beta, \epsilon, j) \geq(1 / 3)^{n} \text { for all } j \geq J_{0} .
$$


This and the definition of $K_{j}$ gives us

$$
\begin{aligned}
\operatorname{vol}\left(K_{j}\right) & =\operatorname{vol}\left(K_{j-1}\right)-\sum_{i=N_{0}+\ldots+N_{j-2}+1}^{N_{0}+\ldots+N_{j-1}} \operatorname{vol}\left(B_{z_{i}}\left(r_{z_{i}}\right)\right) \\
& \leq \operatorname{vol}\left(K_{j-1}\right)-C(H, n, \beta, \epsilon, j-1) \operatorname{vol}\left(K_{j-1}\right) \\
& \leq(1-C(H, n, \beta, \epsilon, j-1)) \operatorname{vol}\left(K_{j-1}\right) \\
& \leq \prod_{k=1}^{j-1}(1-C(H, n, \beta, \epsilon, k)) \operatorname{vol}(K) \\
& \leq\left(1-(1 / 3)^{n}\right)^{\left(j-J_{0}\right)} \operatorname{vol}(K) \quad \forall j \geq J_{0} .
\end{aligned}
$$

We must take $J \geq J_{0}$ sufficiently large such that

$$
3^{n}\left(1-(1 / 3)^{n}\right)^{\left(J-J_{0}\right)}<\epsilon .
$$

Let $r_{i}=r_{i}^{\prime}=r_{z_{i}}$ for $z_{i} \in \bigcup_{j=1}^{J-1} S_{j}$ and $r_{i}=2 r_{i}^{\prime}=2 r_{z_{i}}=2 \beta^{J} \epsilon$ for $z_{i} \in S_{J}$ so that

$$
\bigcup_{i=1}^{N_{1}+\ldots+N_{J}} B_{z_{i}}\left(r_{i}\right) \supset K,
$$

but the $r_{i}^{\prime}$ balls are disjoint, giving us

$$
\sum_{i=1}^{N_{1}+\ldots+N_{J}} \operatorname{vol}\left(B_{z_{i}}\left(r_{i}^{\prime}\right)\right) \leq \operatorname{vol}(K) .
$$

Thus, by (3.4) and the definition of the radii, the fact that $B_{z_{i}}\left(r_{i}\right)$ for $i=$ $1, \ldots, N_{1}+\ldots+N_{J-1}$ are disjoint and in $K$, and (3.35), we get

$$
\begin{aligned}
\sum_{i=1}^{N_{1}+\ldots+N_{J}} \operatorname{vol}\left(B_{z_{i}}\left(r_{i}\right)\right) & \leq \sum_{i=1}^{N_{1}+\ldots+N_{J-1}} \operatorname{vol}\left(B_{z_{i}}\left(r_{i}\right)\right)+\sum_{i=N_{1}+\ldots+N_{J-1}+1}^{N_{1}+\ldots+N_{J}} \operatorname{vol}\left(B_{z_{i}}\left(r_{i}\right)\right) \\
& \leq \operatorname{vol}(K)+\frac{V\left(n, H, 2 \beta^{J} \epsilon\right)}{V\left(n, H, \beta^{J} \epsilon\right)} \sum_{i=N_{1}+\ldots+N_{J-1}+1}^{N_{1}+\ldots+N_{J}} \operatorname{vol}\left(B_{z_{i}}\left(r_{i}^{\prime}\right)\right) \\
(3.39) & \leq \operatorname{vol}(K)+\left(\frac{V\left(n, H, 2 \epsilon \beta^{J}\right)}{V\left(n, H, \epsilon \beta^{J}\right)}\right) \operatorname{vol}\left(K_{J}\right) \\
(3.41) & \leq \operatorname{vol}(K)+3^{n}\left(1-(1 / 3)^{n}\right)^{\left(J-J_{0}\right)} \operatorname{vol}(K) \\
(3.42) & \leq\left(1+3^{n}\left(1-(1 / 3)^{n}\right)^{\left(J-J_{0}\right)}\right) \operatorname{vol}(K) \\
(3.43) & \leq(1+\epsilon) \operatorname{vol}(K) .
\end{aligned}
$$

Finally by (3.31), we also have

$$
\begin{aligned}
\sum_{i=1}^{N_{1}+\ldots+N_{J}} \operatorname{vol}\left(B_{z_{i}}\left(r_{i}^{\prime}\right)\right) & =\operatorname{vol}(K)-\operatorname{vol}\left(K_{J+1}\right) \\
& \geq \operatorname{vol}(K)-\left(1-(1 / 3)^{n}\right)^{\left(J+1-J_{0}\right)} \operatorname{vol}(K) \\
& \geq(1-\epsilon) \operatorname{vol}(K)
\end{aligned}
$$


Recall that we can set $\beta=1 / 2$. Then $\lambda(\epsilon, s, n, H)=\epsilon \beta^{J}$, where $J$ is determined in (3.36), (3.30) and (3.29) and $N(\epsilon, s, n, H)=\sum_{j=1}^{J} N_{j}(\epsilon, \beta, s, n, H)$.

We can now complete our construction of the measure.

Proof of Proposition 3.8 . We are given a pointed Gromov Hausdorff converging sequence of spaces $\tilde{B}\left(p_{i}, r_{i}, R_{i}\right) \delta_{i}$ which converge as covers to $\hat{B}(y, r, R)$. We may need to take a further subsequence to get a renormalized limit measure.

First define the renormalized volume functions

$$
\bar{V}_{i}: \tilde{B}\left(p_{i}, r_{i}, R_{i}\right)^{\delta_{i}} \times \mathbb{R} \rightarrow \mathbb{R}, \quad \bar{V}_{i}(\tilde{x}, \rho)=\operatorname{vol}(B(\tilde{x}, \rho)) / \operatorname{vol}\left(\tilde{p}_{i}, r / 10\right),
$$

where $B(\tilde{x}, \rho)$ is defined using $d_{\tilde{B}\left(p_{i}, r_{i}, R_{i}\right)^{\delta_{i}}}$. As in the proof of ChCo2, Theorem 1.6], we will show that these $\bar{V}_{i}$ are uniformly equicontinuous and bounded, but to do so here we must restrict our domain considerably.

Let $\tilde{p}_{x}=g \tilde{p}_{i}$ be closest to $\tilde{x}$, then $d\left(\tilde{p}_{x}, \tilde{x}\right)=d\left(p_{i}, \pi_{i}(\tilde{x})\right) \leq r$ and

$$
\bar{V}_{i}(\tilde{x}, \rho)=\operatorname{vol}(B(\tilde{x}, \rho)) / \operatorname{vol}\left(\tilde{p}_{x}, r / 10\right) .
$$

Uniformly Bounding the $\bar{V}_{i}$ :

Temporarily fix $R_{0}<r$. We have $R_{0}<r_{i}$ eventually, so we can define $K_{R_{0}}^{i}=$ $\left\{\tilde{x} \in \tilde{B}\left(p_{i}, r_{i}, R_{i}\right)^{\delta_{i}}: d\left(p_{i}, \pi_{i}(\tilde{x}) \leq R_{0}\right\}\right.$ and $K_{R_{0}}=\{\hat{x} \in \hat{B}(y, r, R): d(y, \pi(\hat{x})) \leq$ $\left.R_{0}\right\}$ as in (3.18). Note that by the given continuity of the covering maps as $i \rightarrow \infty$, we have

$$
G H \lim _{i \rightarrow \infty}\left(K_{R_{0}}^{i}, \tilde{p}_{i}\right)=K_{R_{0}}
$$

For any $R_{1} \in\left(0, \min \left\{r-R_{0}, R_{0}+\frac{r}{10}\right\}\right)$ and $R_{2} \in\left(R_{1}, R-r\right)$, then for $i$ sufficiently large we have $R_{1}<r_{i}-R_{0}, R_{1}<R_{0}+r / 10<R_{i}-r_{i}, R_{2}+R_{0}<R_{i}$.

We restrict $\bar{V}_{i}(\tilde{x}, \rho)$ to $K_{R_{0}} \times\left[R_{1}, R_{2}\right]$. These functions are nondecreasing for fixed $\tilde{x}$. We can apply this fact and (3.5) in Corollary 3.5 to get a uniform lower bound,

$$
\begin{aligned}
\bar{V}_{i}(\tilde{x}, \rho) & \geq \bar{V}_{i}\left(\tilde{x}, R_{1}\right)=\operatorname{vol}\left(B\left(\tilde{x}, R_{1}\right)\right) / \operatorname{vol}\left(\tilde{p}_{x}, r / 10\right) \\
& \geq V\left(n, H, R_{1}\right) / V\left(n, H, R_{0}+r / 10\right)>0
\end{aligned}
$$

because $R_{1} \leq\left(R_{0}+r / 10\right) \leq R_{i}-r_{i}$ and $R_{1}<r_{i}-R_{0}$ as required by (3.5).

We get a uniform upper bound for $\bar{V}_{i}$ on $K_{R_{0}} \times\left[R_{1}, R_{2}\right]$ by applying containment and (3.3):

$$
\begin{aligned}
\bar{V}_{i}(\tilde{x}, \rho) & \leq \bar{V}_{i}\left(\tilde{x}, R_{2}\right)=\operatorname{vol}\left(B\left(\tilde{x}, R_{2}\right)\right) / \operatorname{vol}\left(\tilde{p}_{x}, r / 10\right) \\
& \leq \operatorname{vol}\left(B\left(\tilde{p}_{x}, R_{2}+R_{0}\right)\right) / \operatorname{vol}\left(\tilde{p}_{x}, r / 10\right) \\
& \leq V\left(n, H, R_{2}+R_{0}\right) / V(n, H, r / 10)
\end{aligned}
$$

Equicontinuity of the $\bar{V}_{i}$ :

We now further restrict the domain to $K_{R_{0}} \times\left[R_{1}, R_{2}\right]$ if $R_{2}<r-R_{0}$ so that $\bar{V}_{i}(x, \rho)$ are uniformly continuous in the sense of [GP]; see also [Pe Page 279]. The restriction on $R_{2}$ comes from the trouble with estimating large balls using the intrinsic metric. Again we take $i$ sufficiently large such that $R_{2}<r_{i}-R_{0}$.

Given $\tilde{x}_{i} \in K_{R_{0}}^{i}$ and $\rho_{i} \in\left[R_{1}, R_{2}\right]$, let $d_{\tilde{B}\left(p_{i}, r, R\right)^{\delta}}\left(\tilde{x}_{1}, \tilde{x}_{2}\right)=s<\delta<r$. Then $\rho_{i}<r-d_{M}\left(\pi\left(\tilde{x}_{i}\right), p\right)$ and $\rho_{i}<r<R-2 r<R-r-d\left(\tilde{x}_{1}, \tilde{x}_{2}\right)$, so we can apply 
(3.4) to get the following:

$$
\begin{aligned}
\left|\bar{V}_{i}\left(\tilde{x}_{2}, \rho_{2}\right)-\bar{V}_{i}\left(\tilde{x}_{1}, \rho_{1}\right)\right|=\frac{\left|\operatorname{vol}\left(B\left(\tilde{x}_{2}, \rho_{2}\right)\right)-\operatorname{vol}\left(B\left(\tilde{x}_{1}, \rho_{1}\right)\right)\right|}{\operatorname{vol} B(\tilde{p}, r / 10)} \\
=\frac{\operatorname{vol}\left(B\left(\tilde{x}_{2}, \rho_{2}\right) \backslash B\left(\tilde{x}_{1}, \rho_{1}\right)\right)+\operatorname{vol}\left(B\left(\tilde{x}_{1}, \rho_{1}\right) \backslash B\left(\tilde{x}_{2}, \rho_{2}\right)\right)}{\operatorname{vol} B(\tilde{p}, r / 10)} \\
\leq \quad \frac{\operatorname{vol}\left(A n n_{\tilde{x}_{2}}\left(\rho_{2}, \rho_{1}+s\right)\right)+\operatorname{vol}\left(A n n_{\tilde{x}_{1}}\left(\rho_{1}, \rho_{2}+s\right)\right)}{\operatorname{vol} B(\tilde{p}, r / 10)} \\
=\frac{\operatorname{vol}\left(B\left(\tilde{x}_{2}, \rho_{2}\right)\right)}{\operatorname{vol} B\left(\tilde{p}_{x_{2}}, r / 10\right)}\left(\frac{\operatorname{vol}\left(B\left(\tilde{x}_{2}, \rho_{1}+s\right)\right)}{\operatorname{vol}\left(B\left(\tilde{x}_{2}, \rho_{2}\right)\right)}-1\right) \\
\quad+\frac{\operatorname{vol}\left(B\left(\tilde{x}_{1}, \rho_{1}\right)\right)}{\operatorname{vol} B\left(\tilde{p}_{x_{1}}, r / 10\right)}\left(\frac{\operatorname{vol}\left(B\left(\tilde{x}_{1}, \rho_{2}+s\right)\right)}{\operatorname{vol}\left(B\left(\tilde{x}_{1}, \rho_{1}\right)\right)}-1\right) \\
\leq \frac{V\left(n, H, R_{2}\right)}{V(n, H, r / 10)} \cdot\left(\frac{V\left(n, H, \rho_{1}+s\right)-V\left(n, H, \rho_{2}\right)}{V\left(n, H, \rho_{2}\right)}\right. \\
\left.+\frac{V\left(n, H, \rho_{2}+s\right)-V\left(n, H, \rho_{1}\right)}{V\left(n, H, \rho_{1}\right)}\right) .
\end{aligned}
$$

Here $\operatorname{Ann}_{x}\left(\rho_{1}, \rho_{2}\right)=B_{x}\left(\rho_{2}\right) \backslash B_{x}\left(\rho_{1}\right)$ and is empty if $\rho_{2}<\rho_{1}$. Since $V(n, H, s)$ is continuous in $s$, we know that for any $\epsilon$ we can find $\rho_{1}$ near $\rho_{2}$ and $s$ small enough that this last line is less than $\epsilon$.

This gives us uniform equicontinuity with the restricted $R_{2}<r-R_{0}$. Thus we can apply a generalized version of the Arzela-Ascoli theorem ( $\mathrm{GP},[\mathrm{Pe}$, Page 279, Lemma 1.8]) combined with (3.49) to get a subsequence of the $\bar{V}_{i}$ converging uniformly to a limit function defined on $K_{R_{0}} \times\left[0, r-R_{0}\right]$,

$$
\bar{V}_{\infty}(\hat{x}, s)=\lim _{i \rightarrow \infty} \bar{V}_{i}\left(\tilde{x}_{i}, s\right) \text {. }
$$

Extending the domain of $\bar{V}_{\infty}$ :

Recall the definition of $i \tilde{B}$ in (3.18). Then we have

$$
U=\left\{(\hat{x}, s): \hat{x} \in i \hat{B}(y, r, R), s \leq r-d\left(\hat{x}, \hat{p}_{x}\right)\right\}=\bigcup_{R_{0}<r} K_{R_{0}} \times\left[0, r-R_{0}\right] .
$$

We can extend the definition of $\bar{V}_{\infty}$ by taking a sequence of $R_{0} \rightarrow r$ and diagonalizing the subsequences used to define $\bar{V}_{\infty}$ on each $K_{R_{0}} \times\left[0, r-R_{0}\right]$.

Applying (3.4) of Corollary 3.5 we know that

$$
\frac{\bar{V}_{\infty}\left(\hat{x}, r_{1}\right)}{\bar{V}_{\infty}\left(\hat{x}, r_{2}\right)} \geq \frac{V\left(n, H, r_{1}\right)}{V\left(n, H, r_{2}\right)} \forall r_{2}<r-d\left(\hat{x}, \hat{p}_{x}\right) .
$$

Note that unlike (3.4) we only have this estimate on small balls because we only had equicontinuity on the small balls' volumes. Furthermore we have no estimate for volumes of balls centered on the boundary. This is in strong contrast to [ChCo2. Theorem 1.6].

The Caratheodory Construction:

We will now construct the renormalized limit measure on all of $i \hat{B}(y, r, R)$ using a standard Caratheodory construction as in 2.10 of [Fed] or Method II in Mun. This is different than the construction used by Cheeger-Colding because the balls have variable size, but it agrees with their construction on sets contained in a $K_{R_{0}}$ for $R_{0}<r$. 
We first choose our family of open sets,

$$
F:=\left\{B_{\hat{x}}(s):(\hat{x}, s) \in U\right\},
$$

where $U$ is defined in (3.57). Note that these balls can be measured using $d_{\hat{B}(y, r, R)}$ without any difficulties involving the boundary.

For any $A \subset i \hat{B}(y, r, R)$, let

$$
G_{\epsilon, A}=\left\{S \subset F: \bigcup_{B \in S} B \supset A \text { and } \operatorname{diam} B \leq \epsilon \forall B \in S\right\} .
$$

The families of open balls in $G_{\epsilon, A}$ may all be infinite if $A$ is not compact.

Let

$$
\psi: F \rightarrow[0, \infty) \text { be defined } \psi(B(\hat{x}, s))=\bar{V}_{\infty}(\hat{x}, s) .
$$

Then define a measure, $\mu$, as

$$
\mu(A)=\lim _{\epsilon \rightarrow 0} \mu_{\epsilon}(A)
$$

where

$$
\mu_{\epsilon}(A)=\inf _{S \in G_{\epsilon, A}}\left\{\sum_{B \in S} \psi(B)\right\} .
$$

Since all the members of $F$ are Borel sets, $\mu$ is a Borel Regular measure Fed 2.10]. This measure is Radon when restricted to measurable sets by Theorem 13.7 of [Mun] because the members of $F$ are open sets.

Properties of $\mu$ :

If $B(\hat{x}, s) \in F$, then

$$
\mu(B(\hat{x}, s)) \geq \mu_{2 s}(B(\hat{x}, s))=\psi(B(\hat{x}, s))=\bar{V}_{\infty}(\hat{x}, s) .
$$

Now we want to bound $\mu(B(\hat{x}, s))$ from above for arbitrary $\hat{x} \in i \hat{B}(y, r, R)$ and large $s<(R-r) / 2$, where these balls are measured using $d_{\hat{B}(y, r, R)}$ not $d_{i \hat{B}(y, r, R)}$. To avoid trouble, we use the property of Radon measures [Fed Defn. 2.2.5], that

$$
\mu(B(\hat{x}, s))=\sup \{\mu(\bar{K}), \bar{K} \subset B(\hat{x}, s) \text { s.t. } \bar{K} \text { compact }\} .
$$

Note that for any compact $\bar{K}$ in the open set $i \hat{B}(y, r, R)$ there exists $\epsilon=\epsilon_{\bar{K}}>0$ such that $T_{\epsilon}(\bar{K})$ avoids the boundary of $i \hat{B}(y, r, R)$. So in fact $\bar{K} \subset B(\hat{x}, s) \cap K_{R_{0}}$, where $R_{0}=r-\epsilon_{\bar{K}}$ and $K_{R_{0}}$ is defined in (3.18). Thus in fact,

$$
\mu(B(\hat{x}, s))=\sup \left\{\mu\left(B(\hat{x}, s) \cap K_{R_{0}}\right), R_{0}<r\right\} .
$$

Fix $r / 10<R_{0}<r$ and set

$$
K=B(\hat{x}, s) \cap K_{R_{0}} .
$$

Let $\tilde{B}\left(p_{i}, r_{i}, R_{i}\right)^{\delta_{i}}$ be within $\epsilon_{i}<\epsilon_{K} / 10=\left(r-R_{0}\right) / 10$ of $\hat{B}(y, r, R)$, and let

$$
K_{i}=B\left(\tilde{x}_{i}, s+\epsilon_{i}\right) \cap K_{R_{0}+\epsilon_{i}}^{i} \subset \tilde{B}\left(p_{i}, r_{i}, R_{i}\right)^{\delta_{i}} .
$$


Now applying (3.3) as in (3.55), and noting that $s+\epsilon_{i} \leq \frac{R_{i}-r_{i}}{2}$ for all $i$ large, we have

$$
\begin{aligned}
& \frac{\left|\operatorname{vol}\left(K_{i}\right)-\operatorname{vol}\left(B\left(\tilde{x}_{i}, s+\epsilon_{i}\right)\right)\right|}{\operatorname{vol}\left(B\left(\tilde{p}_{i}, r / 10\right)\right)} \\
& \quad \leq \sum_{g \tilde{p}_{i} \in B\left(\tilde{x}_{i}, s+\epsilon_{i}\right)} \operatorname{vol}\left(A n n_{g \tilde{p}_{i}}\left(R_{0}, r_{i}\right)\right) / \operatorname{vol}\left(B\left(g \tilde{p}_{i}, r / 10\right)\right) \\
& \quad=\sum_{g \tilde{p}_{i} \in B\left(\tilde{x}_{i}, s+\epsilon_{i}\right)} \frac{\operatorname{vol}\left(B\left(\tilde{p}_{i}, R_{0}\right)\right)}{\operatorname{vol}\left(B\left(\tilde{p}_{i}, r / 10\right)\right)}\left(\frac{\operatorname{vol}\left(B\left(\tilde{p}_{i}, r_{i}\right)\right)}{\operatorname{vol}\left(B\left(\tilde{p}_{i}, R_{0}\right)\right)}-1\right) \\
& \leq \#\left\{g \tilde{p}_{i} \in B\left(\tilde{x}_{i}, s+\epsilon_{i}\right)\right\} \frac{V(n, H, r)-V\left(n, H, R_{0}\right)}{V(n, H, r / 10)}
\end{aligned}
$$

for all $i$ large. By the given isometry of $\delta / 2$ balls of the covering maps and (3.5), $\#\left\{g \tilde{p}_{i} \in B\left(\tilde{x}_{i}, s+\epsilon_{i}\right)\right\} \leq \frac{V\left(n, H, s+\epsilon_{i}\right)}{V(n, H, \delta / 2)} \leq \frac{V(n, H, 2 s)}{V(n, H, \delta / 2)}$.

So the left side of this equation is uniformly bounded for all $i$ large, therefore

$$
\begin{aligned}
& \forall \varepsilon>0, \exists R_{0} \text { sufficiently close to } r \\
& \text { s.t. }\left|\frac{\operatorname{vol}\left(K_{i}\right)}{\operatorname{vol}\left(B\left(\tilde{p}_{i}, r / 10\right)\right)}-\bar{V}_{i}\left(\tilde{x}_{i}, s+\epsilon_{i}\right)\right| \leq \varepsilon \frac{V(n, H, 2 s)}{V(n, H, \delta / 2)}
\end{aligned}
$$

for all $i$ sufficiently large.

By Lemma 3.11, for all $\epsilon>0$, there exists $\lambda$ and $N$ depending only on $\epsilon, s+\epsilon_{K}$, $n$ and $H$ such that $K_{i}$ has two special families of balls, $H_{i}$ and $\bar{H}_{i}$ consisting of at most $N$ balls each. These balls have of radii between $\epsilon$ and $\lambda$ and satisfy

$$
T_{\epsilon}\left(K_{i}\right) \supset \bigcup_{B \in H_{i}} B \supset K_{i} \text { and } \sum_{B \in H_{i}} \operatorname{vol}(B) \leq(1+\epsilon) \operatorname{vol}\left(K_{i}\right)
$$

and $B$ in $\bar{H}_{i}$ are disjoint subsets of $K_{i}$ such that

$$
\sum_{B \in \bar{H}_{i}} \operatorname{vol}(B) \geq(1-\epsilon) \operatorname{vol}\left(K_{i}\right)
$$

Taking $\epsilon<\epsilon_{K} / 10$ guarantees that if $B\left(\tilde{x}^{\prime}, s^{\prime}\right) \in H_{i}$, then there exists $R_{0}<r$ such that $\left(\tilde{x}^{\prime}, s^{\prime}\right) \in K_{R_{0}}^{i} \times\left[0, r-R_{0}\right]$. Thus

$$
\operatorname{vol}\left(B\left(\tilde{x}^{\prime}, s^{\prime}\right)\right)=\bar{V}_{i}\left(\tilde{x}^{\prime}, s^{\prime}\right) \operatorname{vol}\left(B\left(p_{i}, r / 10\right)\right) \quad \forall\left(\tilde{x}^{\prime}, s^{\prime}\right) \in U .
$$

Let $\Phi_{i}: \tilde{B}\left(p_{i}, r_{i}, R_{i}\right)^{\delta_{i}} \rightarrow \hat{B}(y, r, R)$ be the $\epsilon_{i}$ Hausdorff approximation.

Let $F_{i}^{\prime}=\left\{B_{\Phi_{i}\left(\tilde{x}^{\prime}\right)}\left(s^{\prime}+2 \epsilon_{i}\right): B\left(\tilde{x}^{\prime}, s^{\prime}\right) \subset H_{i}\right\}$. By the choice of $\epsilon$ and $\epsilon_{i}$, we know that $F_{i}^{\prime} \subset F$. In fact if $B\left(\hat{x}^{\prime}, s^{\prime}\right) \in F_{i}^{\prime}$, then $\left(\hat{x}^{\prime}, s^{\prime}\right) \in K_{r-\epsilon} \times[\lambda, \epsilon]$. Furthermore for each $i$ sufficiently large,

$$
K \subset \bigcup_{B \in F_{i}^{\prime}} B .
$$


Thus by (3.63) and the uniform convergence of $\bar{V}_{i}$ there exists $\bar{\epsilon}_{i} \rightarrow 0$,

$$
\begin{aligned}
\mu_{\epsilon+2 \epsilon_{i}}(K) & \leq \sum_{B\left(\hat{x}^{\prime}, s^{\prime}\right) \in F_{i}^{\prime}} \bar{V}_{\infty}\left(\hat{x}^{\prime}, s^{\prime}\right) \\
& \leq \sum_{B\left(\tilde{x}^{\prime}, s^{\prime}\right) \in H_{i}} \bar{V}_{i}\left(\tilde{x}^{\prime}, s^{\prime}\right)+N \bar{\epsilon}_{i} \\
& =\sum_{B\left(\tilde{x}^{\prime}, s^{\prime}\right) \in H_{i}} \operatorname{vol}\left(B\left(\tilde{x}^{\prime}, s^{\prime}\right)\right) / \operatorname{vol}\left(B\left(\tilde{p}_{i}, r / 10\right)\right)+N \bar{\epsilon}_{i} \\
& \leq(1+\epsilon) \operatorname{vol}\left(K_{i}\right) / \operatorname{vol}\left(B\left(\tilde{p}_{i}, r / 10\right)\right)+N \bar{\epsilon}_{i} .
\end{aligned}
$$

Applying our estimate on the volume of $K_{i}$ in (3.70), we get

$$
\mu_{\epsilon+2 \epsilon_{i}}(K) \leq(1+\epsilon)\left(\bar{V}_{i}\left(\tilde{x}_{i}, s+\epsilon_{i}\right)+\varepsilon \frac{V(n, H, 2 s)}{V(n, H, \delta / 2)}\right)+N \bar{\epsilon}_{i} .
$$

We will apply this equation for large and small $s$.

First we look at small $s<r-d(\hat{x}, g \hat{p})$. Since $N$ depends on $\epsilon$ but not on $i$, taking $i$ to infinity, $\epsilon_{i}, \bar{\epsilon}_{i}$ go to 0 , and we get

$$
\mu_{\epsilon}(K) \leq(1+\epsilon)\left(\bar{V}_{\infty}(\hat{x}, s)+\varepsilon \frac{V(n, H, 2 s)}{V(n, H, \delta / 2)}\right) .
$$

Then taking $\epsilon$ to zero, we get

$$
\mu(K) \leq \bar{V}_{\infty}(\hat{x}, s)+\varepsilon \frac{V(n, H, 2 s)}{V(n, H, \delta / 2)} .
$$

Taking the supremum over $K=B(\hat{x}, s) \cap K_{R_{0}}$, using (3.66) with $R_{0} \rightarrow r$ and (3.67), and then taking $\varepsilon$ to zero as in (3.70), we get

$$
\mu(B(\hat{x}, s)) \leq \bar{V}_{\infty}(\hat{x}, s) .
$$

Combining this with (3.64), we get

$$
\mu(B(\hat{x}, s))=\bar{V}_{\infty}(\hat{x}, s), \quad \forall B(\hat{x}, s) \in i \hat{B}(y, r, R),
$$

which gives us (3.12).

To examine $s<(R-r) / 2$, we use (3.79) again. We apply the volume comparison for $s$, to get for $r_{1}<r_{i}-d\left(\tilde{x}_{i}, g \tilde{p}_{i}\right), r_{1}<s$,

$$
\mu_{\epsilon+2 \epsilon_{i}}(K) \leq(1+\epsilon)\left(\frac{V\left(n, H, s+\epsilon_{i}\right)}{V\left(n, H, r_{1}\right)} \bar{V}_{i}\left(\tilde{x}_{i}, r_{1}\right)+\varepsilon \frac{V(n, H, 2 s)}{V(n, H, \delta / 2)}\right)+N \bar{\epsilon}_{i} .
$$

Now taking $i$ to infinity and $\epsilon_{i}, \bar{\epsilon}_{i}$ to 0 , and lastly $\epsilon$ to zero, we get

$$
\mu(K) \leq \frac{V(n, H, s)}{V\left(n, H, r_{1}\right)} \bar{V}_{\infty}\left(\hat{x}, r_{1}\right)+\varepsilon \frac{V(n, H, 2 s)}{V(n, H, \delta / 2)} .
$$

Taking the supremum over $K=K_{R_{0}} \cap B(\hat{x}, s)$ with $R_{0} \rightarrow r$ as in (3.66) and (3.67), and finally taking $\varepsilon$ to zero as in 3.70 , we get

$$
\mu(B(\hat{x}, s)) \leq \frac{V(n, H, s)}{V\left(n, H, r_{1}\right)} \mu\left(B\left(\hat{x}, r_{1}\right)\right)
$$

which gives us (3.13).

We now define $\mu$ as a measure on $\hat{B}(y, r, R)$, by setting $\mu(A)=\mu(A \cap i \hat{B}(y, r, R))$ for any Borel set $A$. Then (3.83) implies (3.12) and (3.86) implies (3.13) for this $\mu$ and $\mu$ is still Borel regular. 
3.3. Stability. We can now use the limit covers $B(y, r, R)^{\delta}$ and their measures (Theorem [2.9] and Proposition 3.8) to get the stability required by Theorem 2.5 to prove the existence of a universal cover for $Y$ (Theorem 1.1).

Theorem 3.12. For all $R>r>0, y \in Y$ there exists $\delta_{y, r, R}$ depending on $Y, y, r, R$ such that for all $\delta<\delta_{y, r, R}$, we have

$$
\tilde{B}(y, r, R)^{\delta}=B(y, r, R)^{\delta}=\tilde{B}(y, r, R)^{\delta_{y, r, R}} .
$$

Note there is no restriction on $R$ and $r$ in this statement.

To prove this we will first prove that for special regular points $y \in Y$ (which are proven to be dense in $Y$ by Cheeger-Colding), sufficiently small balls lift isometrically to all covers. Recall that a regular point is a point in a metric space whose tangent cone is Euclidean and therefore has a pole.

Theorem 3.13. Let $(Y, p)$ be the pointed Gromov-Hausdorff limit of a sequence of complete manifolds $\left(M_{i}^{n}, p_{i}\right)$ such that

$$
\text { Ric }_{M_{i}} \geq-(n-1) H \text { where } n \geq 3, H>0 .
$$

If $y \in B(p, 1) \subset Y$ is a point such that there exists a tangent cone, $\left(Y^{\infty}, y_{\infty}\right)$, that has a pole at $y_{\infty}$, then for any $100 \leq 10 \bar{r} \leq \bar{R}$ there exists $r_{y}(\bar{r}, \bar{R})>0$, such that for all $\delta>0, B\left(y, r_{y}\right)$ lifts isometrically to $B(p, \bar{r}, \bar{R})^{\delta}$.

The proof of Theorem 3.13 uses the Abresch-Gromoll Excess estimate on the relative $\delta$ cover as in [SoWei] Thm 4.5] except that now our covers have boundary. In particular, we need the following adaption of the Abresch Gromoll Excess Theorem AbGl for manifolds with boundary.

Lemma 3.14. Let $M^{n}$ be a compact Riemannian manifold with boundary satisfying (3.88). For a ball $B(p, 10 \rho) \subset M$ not intersecting $\partial M$, there exists a constant

$$
S=S_{n, H}=\min \left\{\frac{1}{8}, \frac{1}{4 \cdot 3^{n}} \frac{1}{\cosh (\sqrt{H} / 4)} \frac{n}{n-1}\left(\frac{n-2}{n-1}\right)^{n-1}\left(\frac{\sqrt{H}}{\sinh \sqrt{H}}\right)^{n-1}\right\}
$$

such that if $\gamma \subset B(p, \rho)$ is a length minimizing curve of $M$ with length $D \leq 1$ and $x \in B(p, \rho)$ satisfying

$$
d_{M}(x, \gamma(0)) \geq\left(S_{n, H}+1 / 2\right) D \quad \text { and } \quad d_{M}(x, \gamma(D)) \geq\left(S_{n, H}+1 / 2\right) D,
$$

then

$$
d_{M}(x, \gamma(D / 2)) \geq 3 S_{n, H} D .
$$

This lemma holds as in the proof of Lemma 4.6 in [SoWei] except for a small technicality involving the use of intrinsic versus restricted metrics. To overcome this, one notes that $d_{M}(x, \gamma(0)) \leq 2 \rho$, so

$$
B_{d_{M}}(x, 2 \rho) \subset B_{d_{M}}(\gamma(0), 4 \rho) \subset B_{d_{M}}(p, 5 \rho) .
$$

By Lemma 2.2 $d_{M}$ and $d_{B(p, 10 \rho)}$ restricted to $B(p, 5 \rho)$ are the same.

Proof of Theorem 3.13. Since $y$ is close enough to $p$, all the points and curves involved in the proof of [SoWei, Theorem 4.5] lie in $B(p, 4.8)$, far away from the boundary $\partial B(p, \bar{r})$, and similarly for the cover. By Lemma 2.2. the restricted distance on $B(p, 4.8)$ from $B(p, \bar{r})$ and from $Y$ are same. So the proof of SoWei] Theorem 4.5] carries over. 
Assume on the contrary that for all $r>0$ there is a $\delta_{r}>0$ such that the ball $B(y, r)$ does not lift isometrically to $B(p, \bar{r}, \bar{R})^{\delta_{r}}$. Let $G^{\delta}$ denote the deck transformation group on $B(p, \bar{r}, \bar{R})^{\delta}$. Thus, there exist $r_{i} \rightarrow 0, \delta_{i}=\delta_{r_{i}}$, and $g_{i} \in G^{\delta_{i}}$ such that $d_{i}=d_{B(p, \bar{r}, \bar{R})^{\delta_{i}}}\left(\tilde{y}, g_{i} \tilde{y}\right) \in\left(0,2 r_{i}\right) \subset(0,1]$. In fact, we can choose $g_{i}$ so that

$$
d_{B(p, \bar{r}, \bar{R})^{\delta_{i}}}\left(\tilde{y}, g_{i} \tilde{y}\right) \leq d_{B(p, \bar{r}, \bar{R})^{\delta_{i}}}(\tilde{y}, h \tilde{y}) \quad \forall h \in G^{\delta_{i}}
$$

Next we will find a length minimizing curve, $\tilde{C}_{i}$, running from $\tilde{y}$ to $g_{i} \tilde{y}$ which has the property that it passes through a particular point $\tilde{z}_{i}=\tilde{C}_{i}\left(d_{i} / 2\right)$ which is the limit of halfway points of length minimizing curves in the sequence $\tilde{B}\left(p_{i}, \bar{r}_{i}, \bar{R}_{i}\right)^{\delta_{i}}$. We do this so that we can apply Lemma 3.14 to $\tilde{B}\left(p_{i}, \bar{r}_{i}, \bar{R}_{i}\right)^{\delta_{i}}$.

To construct $\tilde{C}_{i}$, we first let $\tilde{y}_{j}, \tilde{y}_{j}^{i} \in B\left(\tilde{p_{j}}, 2.4\right) \subset \tilde{B}\left(p_{j}, \bar{r}_{j}, \bar{R}_{j}\right)^{\delta_{i}}$ which are close to $\tilde{y}$ and $g_{i} \tilde{y}$. So $d_{\tilde{B}\left(p_{j}, \bar{r}_{j}, \bar{R}_{j}\right)^{\delta_{i}}}\left(\tilde{y}_{j}, \tilde{y}_{j}^{i}\right)=d_{i, j}$ converges to $d_{i}$. Let $\tilde{z}_{j}^{i}$ be midpoints of minimal geodesics $\gamma_{j}^{i}$, running from $\tilde{y}_{j}$ to $\tilde{y}_{j}^{i}$. Taking a subsequence of $j \rightarrow \infty$, there is a point $\tilde{z}_{i} \in B(p, \bar{r}, \bar{R})^{\delta_{i}}$ which is halfway between $\tilde{y}$ to $g_{i} \tilde{y}$. Let $\tilde{C}_{i}$ be a length minimizing curve running from $\tilde{y}$ to $\tilde{z}_{i}$ and then to $g_{i} \tilde{y}$. Finally let $C_{i}$ be the projection of $\tilde{C}_{i}$ to $B(p, \bar{r})$. $C_{i}$ lies in $B(p, 4.8)$.

Now, imitating the proof of the Halfway Lemma of [So1, and using (3.91), we know $C_{i} \in B(p, 4.8)$ is minimizing halfway around, $d_{Y}\left(C_{i}(0), C_{i}\left(d_{i} / 2\right)\right)=d_{i} / 2$.

We choose a subsequence of these $i$ such that $(Y, y)$ rescaled by $d_{i}$ converges to a tangent cone $\left(Y^{\infty}, y_{\infty}\right)$. So

$$
d_{G H}\left(B\left(y, 10 d_{i}\right) \subset Y, B\left(y_{\infty}, 10 d_{i}\right)\right)<\epsilon_{i} d_{i}
$$

where $\epsilon_{i}$ converges to 0 .

Let $S$ be the constant from Lemma 3.14 Since $Y^{\infty}$ has a pole at $y_{\infty}$, we know there is a length minimizing curve running from $y_{\infty}$ through any point in $\partial B\left(y_{\infty}, d_{i} / 2\right)$ to $\partial B\left(y_{\infty}, d_{i} / 2+2 S d_{i}\right)$. Thus by (3.92),

$$
\forall x \in \partial B\left(y, d_{i} / 2+2 S d_{i}\right) \subset Y,
$$

we have points

$$
x_{\infty} \in A n n_{y_{\infty}}\left(d_{i} / 2+2 S d_{i}-\epsilon_{i} d_{i}, d_{i} / 2+2 S d_{i}+\epsilon_{i} d_{i}\right)
$$

and

$$
y_{i} \in A n n_{y_{\infty}}\left(d_{i} / 2-\epsilon_{i} d_{i}, d_{i} / 2+\epsilon_{i} d_{i}\right)
$$

such that

$$
\begin{aligned}
d_{Y}\left(x, C_{i}\left(d_{i} / 2\right)\right) & <d_{Y^{\infty}}\left(x_{\infty}, y_{i}\right)+\epsilon_{i} d_{i} \\
& \leq 2 \epsilon_{i} d_{i}+2 S d_{i}+\epsilon_{i} d_{i} .
\end{aligned}
$$

Now we will imitate the Uniform Cut Lemma of [So], to show that for all $x \in$ $\partial B\left(y, d_{i} / 2+2 S d_{i}\right)$, we have $l_{i}=d_{Y}\left(x, C_{i}\left(d_{i} / 2\right)\right) \geq(3 S) d_{i}$. This will provide a contradiction for $\epsilon_{i}<S / 2$ and we are done.

First we lift our points $x$ and $y$ to the cover $B(p, \bar{r}, \bar{R})^{\delta_{i}}$ as follows. We lift $y$ to the point $\tilde{y}$ and we lift the closed loop $C_{i}$ to the curve $\tilde{C}_{i}$ running from $\tilde{y}$ through $z_{i}=C_{i}\left(\tilde{d}_{i} / 2\right)$ to $g_{i} \tilde{y}$. Then if $\sigma$ is a length minimizing curve of length $l_{i}$ running from $C_{i}\left(d_{i} / 2\right)$ to $x$, we lift it to $\tilde{Y}^{\delta_{i}}$, so it runs from $\tilde{z}_{i}$ to a new point, $\tilde{x}$. Note that 
by our choice of $x$ in (3.93),

$$
d_{\tilde{Y}^{\delta_{i}}}\left(g_{i} \tilde{y}, \tilde{x}\right) \geq d_{Y}(y, x)=d_{i} / 2+2 S d_{i}
$$

and so is $d_{\tilde{Y} \delta_{i}}(\tilde{y}, \tilde{x})$.

By our choice of $\tilde{C}_{i}$ and $\tilde{z}_{i}$, we know that there are corresponding points in $\tilde{B}\left(p_{j}, \bar{r}_{i}, \bar{R}_{j}\right)^{\delta_{i}}$. That is, there is a triangle formed by $\tilde{y}_{j}, \tilde{y}_{j}^{i}$, with a minimal geodesic $\gamma_{j}^{i}$ running between them and some point $\tilde{x}_{j}$ such that

$$
\begin{aligned}
d_{i, j}=d_{\tilde{B}\left(p_{j}, \bar{r}_{j}, \bar{R}_{j}\right)^{\delta_{i}}}\left(\tilde{y}_{j}, \tilde{y}_{j}^{i}\right) & \rightarrow d_{i}, \\
d_{\tilde{B}\left(p_{j}, \bar{r}_{j}, \bar{R}_{j}\right)^{\delta_{i}}}\left(\tilde{y}_{j}, \tilde{x}_{j}\right) & \rightarrow d_{B(p, \bar{r}, \bar{R})^{\delta_{i}}}(\tilde{y}, \tilde{x})=(1 / 2+2 S) d_{i}, \\
d_{\tilde{B}\left(p_{j}, \bar{r}_{j}, \bar{R}_{j}\right)^{\delta_{i}}}\left(\tilde{y}_{j}^{i}, \tilde{x}_{j}\right) & \rightarrow d_{B(p, \bar{r}, \bar{R})^{\delta_{i}}}\left(g_{i} \tilde{y}, \tilde{x}\right)=(1 / 2+2 S) d_{i}, \\
l_{i, j}=d_{\tilde{B}\left(p_{j}, \bar{r}_{j}, \bar{R}_{j}\right)^{\delta_{i}}}\left(\tilde{\gamma}_{j}^{i}\left(d_{i, j} / 2\right), \tilde{x}_{j}\right) & \rightarrow d_{B(p, \bar{r}, \bar{R})^{\delta_{i}}}\left(\tilde{z}_{i}, \tilde{x}\right)=l_{i} .
\end{aligned}
$$

So for $j$ sufficiently large, we have

$$
d_{\tilde{B}\left(p_{j}, \bar{r}_{j}, \bar{R}_{j}\right)^{\delta_{i}}}\left(\tilde{y}_{j}, \tilde{x}_{j}\right) \geq(1 / 2+S) d_{i, j} \text { and } d_{\tilde{B}\left(p_{j}, \bar{r}_{j}, \bar{R}_{j}\right)^{\delta_{i}}}\left(\tilde{y}_{j}^{i}, \tilde{x}_{j}\right) \geq(1 / 2+S) d_{i, j}
$$

and can apply Lemma 3.14 to get

$$
l_{i, j} \geq 3 S d_{i, j} .
$$

Taking $j$ to infinity, we get the limit of this bound in $B(p, \bar{r}, \bar{R})^{\delta_{i}}$, namely $l_{i} \geq$ $3 S d_{i}$. This contradicts (3.96) for $\epsilon_{i}<S / 2$ and we are done.

We can now prove our stability theorem. We first state a more geometrically intuitive theorem and then prove that it implies Theorem 3.12

Theorem 3.15. For all $R>0, y \in Y$ one of the following two statements holds:

I. There exists $\delta_{y, R}$ depending on $Y, y, R$ such that for all $\delta<\delta_{y, R}$, we have

$$
\tilde{B}(y, R)^{\delta}=\tilde{B}(y, R)^{\delta_{y, R}} .
$$

II. For all $R^{\prime}<R$ there exists $\delta_{R^{\prime}}$ depending on $Y, y, R, R^{\prime}$ such that

$$
\tilde{B}\left(y, R^{\prime}, R\right)^{\delta_{R^{\prime}}}=\tilde{B}\left(y, R^{\prime}, R\right)^{\delta} \quad \forall \delta<\delta_{R^{\prime}} .
$$

Note that Theorem 3.15 is essentially saying that if there is a problem with arbitrarily small noncontractible curves, then they are near the boundary.

Proof of Theorem 3.12, For a given $y, r$ and $R$, we apply Theorem 3.15 to $B(y, R)$. If case I holds, then taking $\delta_{y, r, R}=\delta_{y, R}$ we have $B(y, r, R)^{\delta}=B(y, r, R)^{\delta_{y, r, R}}$ for any $r<R$. If case II holds, we let $R^{\prime}=r$ and $\delta_{y, r, R}=\delta_{R^{\prime}}$. Then (3.87) follows from (2.9) of Theorem 2.9.

Proof of Theorem 3.15. Suppose neither I nor II hold. Then there exists $y$ and $R$ and $\delta_{i}$ converging to 0 such that $\tilde{B}(y, R)^{\delta_{i}}$ are all distinct. Then there exists a sequence of $\delta_{i}>0$ with $\delta_{1} \leq R / 10, \delta_{i}>10 \delta_{i+1}$ such that all $\tilde{B}(y, R)^{\delta_{i}}$ and $G\left(y, R, \delta_{i}\right)$ are distinct. In particular there are nontrivial elements of $G\left(y, R, \delta_{i}\right)$ which are trivial in $G\left(y, R, \delta_{i-1}\right)$. So there exist $x_{i} \in B(y, R)$ such that the $B_{x_{i}}\left(\delta_{i-1}\right)$ contains a noncontractible loop, $C_{i}$, which lifts nontrivially in $\tilde{B}(y, R)^{\delta_{i}}$.

In fact we can choose $x_{1}$ to be the point closest to $y$ such that $B_{x_{1}}(R / 10)$ contains a noncontractiblle loop and then choose $\delta_{1} \in(0, R / 10]$ as small as possible such that $B_{x_{1}}(R / 10)$ contains a loop $C_{1}$ which lifts nontrivially to $\tilde{B}(y, R)^{\delta_{1}}$. We can then choose iteratively $x_{j}$ the point closest to $y$ such that $B_{x_{j}}\left(\delta_{j-1} / 10\right)$ contains a 
noncontractible loop. Then set $\delta_{j} \in\left(0, \delta_{j-1} / 10\right]$ as small as possible so that $B_{x_{j}}\left(\delta_{j}\right)$ contains a loop $C_{j}$ which lifts nontrivially to $\tilde{B}(y, R)^{\delta_{j}}$. Note that $d_{B(y, R)}\left(y, x_{j}\right)$ is a nondecreasing sequence.

By compactness, a subsequence of the $x_{i}$ converge to some point $x$ in $B(y, R)$.

If $x \in \partial B(y, R)$, then for any $R^{\prime}<R$, we know that there exists $N_{1}$ sufficiently large such that $d_{B(y, R)}\left(y, x_{j}\right)>R^{\prime}+\left(R-R^{\prime}\right) / 2$ for all $j \geq N_{1}$. There exists $N_{2}>N_{1}$ such that $\delta_{(j-1) / 10}<\left(R-R^{\prime}\right) / 2$ for all $j \geq N_{2}$.

By the choice of our sequence of $x_{j}$, this implies that if $C$ is a loop contained in $B\left(y^{\prime}, \delta\right)$ where $\delta \leq \delta_{\left(N_{2}-1\right) / 10}$ and $B\left(y^{\prime}, \delta\right) \cap B\left(y, R^{\prime}\right)$ is nonempty, then $C$ is contractible in $B(y, R)$. Thus

$$
\tilde{B}\left(y, R^{\prime}, R\right)^{\delta_{R^{\prime}}}=\tilde{B}\left(y, R^{\prime}, R\right)^{\delta} \quad \forall \delta<\delta_{\left(N_{2}-1\right) / 10}=\delta_{R^{\prime}} .
$$

This implies Case II which we have assumed to be false.

So now we know $x$ is not in the boundary of $B(y, R)$, and we proceed to find a contradiction.

Let $\bar{R}>0$ be defined such that $B(x, \bar{R}) \subset B(y, R)$ and let $\bar{r}=\bar{R} / 10>0$. Eventually the $C_{i}$ is in $B(x, \bar{r} / 6)$.

Note $C_{i} \in B_{x_{i}}\left(\delta_{i-1}\right)$ so they lift as closed curves to $\tilde{B}(x, \bar{r}, \bar{R})^{\delta_{i-1}}$. Since they lift nontrivially to $\tilde{B}(y, R)^{\delta_{i}}, C_{i}$ also lifts nontrivially to $\tilde{B}(x, \bar{r}, \bar{R})^{\delta_{i}}$.

Since $C_{i}$ must lift to a union of balls $B_{g \tilde{x}_{i}}\left(\delta_{i-1}\right)$ in $\tilde{B}(x, \bar{r}, \bar{R})^{\delta_{i}}$, there exists $g_{i}$ nontrivial in $G\left(x, \bar{r}, \bar{R}, \delta_{i}\right)$ such that

$$
d_{\tilde{B}_{i}^{\delta}}\left(g_{i} \tilde{x}_{i}, \tilde{x}_{i}\right)<2 \delta_{i-1} .
$$

Let $\alpha_{i}$ be the projection of the minimal curve from $g_{i} \tilde{x}_{i}$ to $\tilde{x}_{i}$. Then $L\left(\alpha_{i}\right)<$ $2 \delta_{i-1}<\bar{r}$ and

$$
\alpha_{i} \subset B_{x_{i}}\left(2 \delta_{i-1}\right) \subset B\left(x, \bar{r} / 6+2 \delta_{i}\right) \subset B(x, \bar{r} / 3) .
$$

The $\alpha_{i}$ represents an element $g_{i}$ of $\pi_{1}(B(x, \bar{r}))$ which is mapped nontrivially into $G\left(x, \bar{r}, \bar{R}, \delta_{i}\right)$ and trivially into $G\left(x, \bar{r}, \bar{R}, 2 \delta_{i-1}\right)$.

For any $j$, the limit cover $B(x, \bar{r}, \bar{R})^{\delta_{j}}$ covers $B(x, \bar{r}, \bar{R})^{\delta_{i}}$ for $i=1, \ldots, j-1$. By Theorem 2.9 $g_{1}, \ldots, g_{j-1}$ are distinct nontrivial deck transforms of $B(x, \bar{r}, \bar{R})^{\delta_{j}}$.

Furthermore, for any $q \in B(x, \bar{r})$, letting $\tilde{x}_{i}$ be the lift of $x_{i}$ closest to $\tilde{q} \in$ $B(x, \bar{r}, \bar{R})^{\delta_{j}}$, we have,

$$
\begin{aligned}
& d_{B(x, \bar{r}, \bar{R})^{\delta_{j}}}\left(\tilde{q}, g_{i} \tilde{q}\right) \leq d_{B(x, \bar{r}, \bar{R})^{\delta_{j}}}\left(\tilde{q}, \tilde{x}_{i}\right)+d_{B(x, \bar{r}, \bar{R})^{\delta_{j}}}\left(\tilde{x}_{i}, g_{i} \tilde{x}_{i}\right) \\
& +d_{B(x, \bar{r}, \bar{R})^{\delta_{j}}}\left(g_{i} \tilde{x}_{i}, g_{i} \tilde{q}\right) \\
& \leq 2 \bar{r}+2 L\left(\alpha_{i}\right)+2 \bar{r} \leq 6 \bar{r} \text {. }
\end{aligned}
$$

So the $\delta_{j}$-length of $g_{i}$, defined in Definition 2.7 is

$$
l\left(g_{i}, \delta_{j}\right)=\inf _{q \in B(x, \bar{r})} d_{B(x, \bar{r}, \bar{R})^{\delta_{j}}}\left(\tilde{q}, g_{i} \tilde{q}\right) \leq 6 \bar{r} .
$$

Therefore we have for any $j$, there are $j-1$ distinct elements in $G(x, \bar{r}, \bar{R})^{\delta_{j}}$ with $l\left(g_{i}, \delta_{j}\right) \leq 6 \bar{r}$.

On the other hand we claim that the total number of elements in $G(x, \bar{r}, \bar{R})^{\delta}$ of $\delta$-length $\leq 6 \bar{r}$ is uniformly bounded for all $\delta$ in terms of geometry and topology of $B(x, \bar{r})$.

To show this claim, let us look at the lift of a regular point $p \in B(x, \bar{r})$ in the cover $B(x, \bar{r}, \bar{R})^{\delta / 2}$. 
We know that by Theorem 3.13 there is a $\delta_{p}>0$ such that the ball of radius $\delta_{p}$ about $p$ is isometrically lifted to disjoint balls of radius $\delta_{p}$ in $B(x, \bar{r}, \bar{R})^{\delta}$. Let

$$
\delta_{0}=\min \left\{\delta_{p}, \bar{r}\right\} .
$$

Let $N$ be the number of distinct elements in $G(x, \bar{r}, \bar{R})^{\delta}$ of $\delta$-length $\leq 6 \bar{r}$. Note that $g B\left(\tilde{p}, \delta_{0}\right)$ is contained in $B\left(\tilde{p}, 6 \bar{r}+\delta_{0}\right) \subset \tilde{B}(x, \bar{r}, \bar{R})^{\delta}$ for all $g \in G(x, \bar{r}, \bar{R})^{\delta}$ with $l(g, \delta) \leq 6 \bar{r}$

Now we cannot control $\tilde{B}(x, \bar{r}, \bar{R})^{\delta}$ very well because it does not have a Bishop Gromov volume comparison theorem. However in Theorem 3.8 we showed there is a good measure, $\mu$, on the limit cover $B(x, \bar{r}, \bar{R})^{\delta / 2}$, which is a cover of $\tilde{B}(x, \bar{r}, \bar{R})^{\delta}$ by Theorem 2.9. So we can lift the distinct $g$ by lifting representative minimal curves from $\tilde{p}$ to $g \tilde{p}$. Thus there are $N+1$ isometric disjoint balls of radius $\delta_{0}$ contained in a ball of radius $6 \bar{r}+\delta_{0}$ in the limit cover. Here we have included the center ball as well.

Thus, applying the properties of $\mu$ from Proposition 3.8, we have

$$
N+1 \leq \frac{\mu\left(B\left(\tilde{q}, 6 \bar{r}+\delta_{0}\right)\right)}{\mu\left(B\left(\tilde{q}, \delta_{0}\right)\right)} \leq V\left(n, H, 6 \bar{r}+\delta_{0}\right) / V\left(n, H, \delta_{0}\right)
$$

because $6 \bar{r}+\delta_{0}<\bar{R}-\bar{r}$.

This gives us a contradiction.

We now prove Corollary 3.10 concerning the convergence of measures of sets.

Proof of Corollary [3.10. We must show that given any $\epsilon_{i} \rightarrow 0$,

$$
\lim _{i \rightarrow \infty} \frac{\operatorname{Vol}\left(T_{\epsilon_{i}}\left(K_{i}\right)\right)}{\operatorname{Vol}\left(B\left(\tilde{p}_{i}, r / 10\right)\right)}=\mu(K) .
$$

Since $K \subset \operatorname{int}(X)$ is compact, there is an $\epsilon_{0}>0$ such that $T_{4 \epsilon_{0}}(K) \subset \operatorname{int}(X)$ and so we can use the Cheeger-Colding Caratheodory method to define the measure.

Given any $V>0$ and $\epsilon<\epsilon_{0}$, there exist $z_{1}, \ldots, z_{n} \in K$ such that $\bigcup_{j} B_{z_{j}}\left(r_{j}\right) \supset K$, $r_{j}<\epsilon$ and

$$
\mu(K) \leq \sum_{j=1}^{n} \mu\left(B_{z_{j}}\left(r_{j}\right)\right) \leq \mu(K)+V .
$$

Thus by the definition of the renormalized limit and the uniform continuity of $\bar{V}_{i}$, there are $z_{j, i} \in K_{i}$ such that

$$
\mu(K) \leq \lim _{i \rightarrow \infty} \sum_{j=1}^{n} \frac{\operatorname{Vol}\left(B_{z_{j i}}\left(r_{j}+2 \epsilon_{i}^{\prime}+\epsilon_{i}\right)\right)}{\operatorname{Vol}\left(B\left(\tilde{p}_{i}, r / 10\right)\right)} \leq \mu(K)+V,
$$

where $\epsilon_{i}^{\prime}$ is the Gromov Hausdorff estimate from $X_{i}$ to $X$ and $\epsilon_{i}$ is any sequence converging to 0 .

In particular, for all $i \geq N_{1}$,

$$
\mu(K)-V \leq \sum_{j=1}^{n} \frac{\operatorname{Vol}\left(B_{z_{j i}}\left(r_{j}\right)\right)}{\operatorname{Vol}\left(B\left(\tilde{p}_{i}, r / 10\right)\right)} \leq \sum_{j=1}^{n} \frac{\operatorname{Vol}\left(B_{z_{j i}}\left(r_{j}+2 \epsilon_{i}^{\prime}+\epsilon_{i}\right)\right)}{\operatorname{Vol}\left(B\left(\tilde{p}_{i}, r / 10\right)\right)} \leq \mu(K)+2 V .
$$

By the choice of $\epsilon_{i}^{\prime}$,

$$
\sum_{j=1}^{n} \operatorname{Vol}\left(B_{z_{j i}}\left(r_{j}+2 \epsilon_{i}^{\prime}+\epsilon_{i}\right)\right) \geq \operatorname{Vol}\left(T_{\epsilon_{i}}\left(K_{i}\right)\right) .
$$


Thus

$$
\limsup _{i \rightarrow \infty} \frac{\operatorname{Vol}\left(T_{\epsilon_{i}}\left(K_{i}\right)\right)}{\operatorname{Vol}\left(B\left(\tilde{p}_{i}, r / 10\right)\right)} \leq \mu(K)+2 V \quad \forall V>0,
$$

so the limsup is $\leq \mu(K)$. On the other hand

$$
\sum_{j=1}^{n} \operatorname{Vol}\left(B_{z_{j i}}\left(r_{j}\right)\right) \leq \operatorname{Vol}\left(T_{\epsilon}\left(K_{i}\right)\right)
$$

So for all $\epsilon>0$

$$
\liminf _{i \rightarrow \infty} \frac{\operatorname{Vol}\left(T_{\epsilon}\left(K_{i}\right)\right)}{\operatorname{Vol}\left(B\left(\tilde{p}_{i}, r / 10\right)\right)} \geq \mu(K)
$$

Therefore we can choose $\epsilon_{i} \rightarrow 0$ such that

$$
\liminf _{i \rightarrow \infty} \frac{\operatorname{Vol}\left(T_{\epsilon_{i}}\left(K_{i}\right)\right)}{\operatorname{Vol}\left(B\left(\tilde{p}_{i}, r / 10\right)\right)} \geq \mu(K)
$$

and using the same $\epsilon_{i}$ in (3.114), we are done.

\section{Properties of $\tilde{Y}$ And its applications}

In this section we first study properties of the universal cover $\tilde{Y}$. We begin by showing that domains in the universal cover are Gromov Hausdorff limits of relative delta covers (Theorem 4.2) and (Corollary 4.3). We then easily show that there is a measure on $\tilde{Y}$ such that Bishop-Gromov's volume comparison holds (Theorem4.5). We then show that the Cheeger-Gromoll Splitting Theorem (Theorem 4.6) holds. Finally we will derive some applications.

Recall, as in Example 1.2, that there may be no covers of the $M_{i}$ that converge to $\tilde{Y}$. The following example demonstrates that a region in $\tilde{Y}$ may not be a limit of relative delta covers with a uniformly bounded outer radius $R$.

Example 4.1. There is a complete noncompact 4-manifold $M$ with sectional curvature constant equal to -1 and its fundamental group is two generated, infinitely presented such that $\operatorname{ker}\left(\pi_{1}(B(p, r)) \rightarrow \pi_{1}(B(p, R))\right)$ is strictly smaller than $\operatorname{ker}\left(\pi_{1}(B(p, r)) \rightarrow \pi_{1}(M, p)\right)$ for all $r \geq 1, R \geq r$ [BoMe], [Po]. So the universal covering of $M$ restricted to $B(p, r)$ and the universal covering of $B(p, R)$ restricted to $B(p, r)$ are always different for all $R \geq r$.

We thank D. Cooper for bringing this and other similar examples to our attention.

One consequence of this example is that we cannot hope to study a ball in $\tilde{Y}$ just by applying Theorem 2.9. This example demonstrates that the lift of a ball $B(y, r)$ to $\tilde{Y}$ may not be isometric to the limit of any relative delta cover no matter how large we take $R$ and how small we take $\delta$. The following theorem allows us to study regions in $\tilde{Y}$ using limits of relative delta covers with $R \rightarrow \infty$.

Theorem 4.2. Given any $\tilde{x} \in \tilde{Y}$ and $0<r<\infty$, let $x=\pi(\tilde{x}) \in Y$ and $\bar{B}(x, r)$ be the connected lift of $B(x, r)$ in $\tilde{Y}$ containing $\tilde{x}$. Each space is given the intrinsic metric. Then $(\bar{B}(x, r), \tilde{x})$ is the pointed Gromov Hausdorff limit of stable relative delta covers $\left(\tilde{B}\left(x, r, R_{i}\right)^{\delta_{i}}=B\left(x, r, R_{i}\right)^{\delta_{i}}, \tilde{x}_{i}\right)$, where $\tilde{x}_{i}$ is a lift of $x_{i}$ and $R_{i}>3 r$ diverge to infinity. Furthermore these relative delta covers all cover $\bar{B}(x, r)$. 
Since each $B\left(x, r, R_{i}\right)^{\delta_{i}}$ is a GH limit of $\tilde{B}\left(x_{j}, r_{j}, R_{i, j}\right)^{\delta_{i}}$ by definition, we have the following immediate corollary.

Corollary 4.3. In fact $(\bar{B}(x, r), \tilde{x})$ is the pointed Gromov Hausdorff limit of a subsequence of relative $\delta$ covers of $M_{i}^{n}$. Namely if we still index the subsequence as $k$, then there are $r_{k} \rightarrow r, R_{k} \rightarrow \infty, B\left(x_{k}, R_{k}\right) \subset M_{k}^{n}$ such that $\left(\tilde{B}\left(x_{k}, r_{k}, R_{k}\right)^{\delta_{k}}, \tilde{x}_{k}\right)$ converges to $(\bar{B}(x, r), \tilde{x})$.

Proof. We have that $(\bar{B}(x, r), \tilde{x})$ is the pointed Gromov Hausdorff limit of $\left(B\left(x, r, R_{i}\right)^{\delta_{i}}, \tilde{x}_{i}\right)$ for some $R_{i} \geq 3 r$ going to infinity and $\delta_{i}$ decreasing. So

$$
d_{G H}\left(B\left(x, r, R_{i}\right)^{\delta_{i}}, \bar{B}(x, r)\right) \leq \epsilon_{i}
$$

for some $\epsilon_{i} \rightarrow 0$. On the other hand each $B\left(x, r, R_{i}\right)^{\delta_{i}}$ is a GH limit of $\tilde{B}\left(x_{j}, r_{j}, R_{i, j}\right)^{\delta_{i}}$. For $B\left(x, r, R_{1}\right)^{\delta_{1}}$, choose $j_{1}$ sufficiently large such that

$$
d_{G H}\left(\tilde{B}\left(x_{j_{1}}, r_{j_{1}}, R_{i, j_{1}}\right)^{\delta_{1}}, B\left(x, r, R_{1}\right)^{\delta_{1}}\right) \leq \epsilon_{1},
$$

and for $B\left(x, r, R_{2}\right)^{\delta_{2}}$, choose $j_{2} \geq j_{1}$ sufficiently large such that

$$
d_{G H}\left(\tilde{B}\left(x_{j_{2}}, r_{j_{2}}, R_{i, j_{2}}\right)^{\delta_{2}}, B\left(x, r, R_{2}\right)^{\delta_{2}}\right) \leq \epsilon_{2} .
$$

Continuing to choose a subsequence in this way, we have

$$
d_{G H}\left(\tilde{B}\left(x_{j_{k}}, r_{j_{k}}, R_{i, j_{k}}\right)^{\delta_{k}}, B\left(x, r, R_{k}\right)^{\delta_{k}}\right) \leq \epsilon_{k} .
$$

Renaming the index $j_{k}$ as $k$ we have $d_{G H}\left(\tilde{B}\left(x_{k}, r_{k}, R_{i, k}\right)^{\delta_{k}}, \bar{B}(x, r)\right) \leq 2 \epsilon_{k}$.

We now prove Theorem 4.2 by showing that regions in the universal cover of the limit space are limits themselves.

Proof of Theorem 4.2. First note that for $R>3 r, \bar{B}(x, r)$ can also be seen as the connected lift of $B(x, r)$ in $\bar{B}(x, R)$ containing $\tilde{x}$.

By the definition of a cover, we know that for all $z \in B(x, R)$, there is a $\delta_{z}>0$ such that $B_{z}\left(\delta_{z}\right)$ lifts isometrically to $\bar{B}(x, R)$. By compactness of $B(x, R)$, there exists a $\delta_{0}>0$ such that any $B_{z}\left(\delta_{0}\right)$ lifts isometrically to the universal cover and thus to $\bar{B}(x, R)$. By [Sp, p. 81] and Definition 2.5, $\tilde{B}(x, R)^{\delta_{0}}$ must cover $\bar{B}(x, R)$.

So $\bar{B}(x, r)$ is covered by $\tilde{B}(x, r, R)^{\delta_{0}}$. Applying Theorem 3.12] we know that there exists $\delta_{R}>0$ such that the relative delta covers stabilize, so for all $\delta<\delta_{R}$, we have that $\tilde{B}(x, r, R)^{\delta}=B(x, r, R)^{\delta}=\tilde{B}(x, r, R)^{\delta_{R}}$ all cover $\bar{B}(x, r)$.

Now let us take a sequence of $R_{i}$ diverging to infinity and let $\delta_{i}$ be chosen such that $\delta_{i} \leq \delta_{R_{i}}$ and $\delta_{i}$ decrease. Then by the stabilization $\tilde{B}\left(x, r, R_{i}\right)^{\delta_{i+1}}=$ $\tilde{B}\left(x, r, R_{i}\right)^{\delta_{i}}$. Furthermore $\tilde{B}\left(x, r, R_{i}\right)^{\delta_{i+1}}$ covers $\tilde{B}\left(x, r, R_{i+1}\right)^{\delta_{i+1}}$ because any curve that lifts to a closed curve in $\tilde{B}\left(x, r, R_{i}\right)^{\delta_{i+1}}$ is homotopic in $B\left(x, R_{i}\right)$ to a combination of curves of the form $\alpha \beta \alpha^{-1}$ with $\beta$ in a $\delta_{i+1}$ ball. This is also true if we allow the homotopy in the larger ball $B\left(x, R_{i+1}\right)$.

Thus we have

$$
\begin{aligned}
\tilde{B}\left(x, r, R_{i}\right)^{\delta_{i}} & =B\left(x, r, R_{i}\right)^{\delta_{i}} \rightarrow \tilde{B}\left(x, r, R_{i+1}\right)^{\delta_{i+1}} \\
& =B\left(x, r, R_{i+1}\right)^{\delta_{i+1}} \rightarrow \cdots \rightarrow \bar{B}(x, r) .
\end{aligned}
$$

Let $\tilde{x}_{i}$ be a lift of $x_{i}$ to $\tilde{B}\left(x, r, R_{i}\right)^{\delta_{i}}$.

We claim that a subsequence of $\left(\tilde{B}\left(x, r, R_{i}\right)^{\delta_{i}}, \tilde{x}_{i}\right)$ converges in the pointed Gromov Hausdorff sense to a limit space $\bar{B}(x, r, \infty)$.

To prove this we apply Gromov's Compactness Theorem [Gr, Page 280, Lemma 1.9], [Pe]. Given any $\rho>0, \epsilon>0$ we must show that for $i$ sufficiently large 
there is a uniform bound on the number of disjoint balls of radius $\epsilon$ contained in a ball $B\left(\tilde{x}_{i}, \rho\right)$. We can just take $i$ large enough that $R_{i}>4 \rho$, and then apply Proposition 3.8 using the fact that $\tilde{B}\left(x, r, R_{i}\right)^{\delta_{i}}=B\left(x, r, R_{i}\right)^{\delta_{i}}$ with renormalized limit measures.

We claim that $\bar{B}(x, r, \infty)$ is a cover of $\bar{B}(x, r)$.

By Lemma 2.3 we know that the covering map $\pi_{1}: \tilde{B}\left(x, r, R_{1}\right)^{\delta_{1}} \rightarrow \bar{B}(x, r)$ is an isometry on balls of radius $\delta_{1} / 3$. Since we have covers $f_{i}: \tilde{B}\left(x, r, R_{1}\right)^{\delta_{1}} \rightarrow$ $\tilde{B}\left(x, r, R_{i}\right)^{\delta_{i}}$ and $\pi_{i}: \tilde{B}\left(x, r, R_{i}\right)^{\delta_{i}} \rightarrow \bar{B}(x, r)$, each $\pi_{i}$ must preserve the same isometry of balls of radius $\delta_{1} / 3$. Furthermore the $\pi_{i}$ are uniformly equicontinuous, so by a generalized Arzela-Ascoli theorem (see, e.g., Pe, Page 279, Lemma 1.8]) a subsequence converges to a continuous function $\pi_{\infty}: \bar{B}(x, r, \infty) \rightarrow \bar{B}(x, r)$ which is an isometry on balls of radius less than $\delta_{1} / 3$. Thus $\pi_{\infty}$ is a covering map.

We claim that $\bar{B}(x, r, \infty)$ is isometric to $\bar{B}(x, r)$.

Since $\bar{B}(x, r, \infty)$ is a cover of $\bar{B}(x, r)$, we need only show that all loops in $B(x, r)$ which lift nonclosed to $\bar{B}(x, r, \infty)$ also lift nonclosed to $\bar{B}(x, r)$ Sp, Page 78, Lemma 9]. But if a loop $C$ lifts nonclosed to $\bar{B}(x, r, \infty)$, then by the Hausdorff approximation it lifts nonclosed to all $B\left(x, r, R_{i}\right)^{\delta_{i}}$ for $i$ sufficiently large depending on $C$. So $C$ is not homotopic in $B\left(x, R_{i}\right)$ to a combination of loops $\alpha \beta \alpha^{-1}$ with $\beta$ in a ball of radius $\delta_{i}$. In particular $C$ is not contractible in $B\left(x, R_{i}\right)$. But this is true for $R_{i}$ arbitrarily large. Thus $C$ must not be contractible. If it were contractible, there would be a homotopy $H: I \times I \rightarrow Y$, but $i m(H)$ would be compact and fit in some $B\left(x, R_{i}\right)$.

Our first application of Theorem 4.2 will be to define a renormalized limit measure on $\tilde{Y}$ and prove that it satisfies the Bishop Gromov Volume comparison globally. We begin with a lemma.

Lemma 4.4. Given a length space $X_{1}$ with a measure $\mu_{1}$ and a covering $\pi_{12}$ : $X_{2} \rightarrow X_{1}$, there is a natural lifted measure $\mu_{2}=\mu_{\pi_{12}}$ on $X_{2}$ so that the covering map $\pi_{12}$ is locally measure preserving and globally measure nonincreasing.

Furthermore, if $X_{3} \rightarrow X_{2} \rightarrow X_{1}$ are all coverings, then $\mu_{3}=\mu_{\pi_{13}}$, where $\pi_{13}$ : $X_{3} \rightarrow X_{1}$ agrees with $\mu_{\pi_{23}}$, the natural lifted measure from $X_{2}$ to $X_{3}$ of $\mu_{\pi_{12}}$.

Proof. We can define a function $\Phi$ on small open balls in $X_{2}$ which are projected isometrically to $X_{1}$ by pulling back the measure on $X_{1}$. Now for any closed subset $A \subset X_{2}$, we define $\bar{\mu}(A)$ by the Caratheodory's construction (see (3.14) and (3.15)). Since the measure on $Y$ was also constructed using Caratheodory based on the same $\Phi$ [ChCo2], the covering map $\pi$ is locally measure preserving. This gives a Borel measure on $X_{2}$. Since the $\Phi$ will agree locally on $X_{3}$ as well, it defines the same lift in two steps or in one.

One can use packing arguments to see that $\pi$ is measure nonincreasing.

Theorem 4.5. The universal cover $\tilde{Y}$ with the natural lifted measure $\tilde{\mu}=\mu_{\pi}$, of the renormalized limit measure $\mu_{Y}$ on $Y$, satisfies Bishop-Gromov volume comparision globally,

$$
\frac{\tilde{\mu}\left(B\left(\tilde{x}, r_{1}\right)\right)}{\tilde{\mu}\left(B\left(\tilde{x}, r_{2}\right)\right)} \geq V\left(n, H, r_{1}\right) / V\left(n, H, r_{2}\right) \quad \forall r_{1} \leq r_{2}, \tilde{x} \in \tilde{Y} .
$$

Proof. By Theorem4.2, we know that for any fixed $r,(\bar{B}(y, r), \tilde{y})$ is the pointed Gromov Hausdorff limit of stable relative delta covers $\left(\tilde{B}\left(y, r, R_{i}\right)^{\delta_{i}}=B\left(y, r, R_{i}\right)^{\delta_{i}}, \tilde{y}_{i}\right)$, 
where $\tilde{y}_{i}$ is a lift of $y$ and $R_{i}$ diverge to infinity. Furthermore these relative delta covers all cover $\bar{B}(y, r)$. In particular by Lemma 2.3 all these relative delta covers act as isometries on balls of radius $\delta_{1} / 4$. Note that $\delta_{1}$ depends on $r$, so we will let $\delta_{r}=\delta_{1} / 2$.

Now each $\left(B\left(y, r, R_{i}\right)^{\delta_{i}}, \tilde{y}_{i}\right)$ is a limit of $\left(\tilde{B}\left(p_{j}, r_{j}, R_{i, j}\right)^{\delta_{i}}, \tilde{p}_{j}\right)$, so for each $i$ we can take $j_{i}$ sufficiently large that

$$
d_{G H}\left(\left(B\left(y, r, R_{i}\right)^{\delta_{i}}, \tilde{y}_{i}\right),\left(\tilde{B}\left(p_{j_{i}}, r_{j_{i}}, R_{i, j_{i}}\right)^{\delta_{i}}, \tilde{p}_{j_{i}}\right)\right)<\epsilon_{i},
$$

where $\epsilon_{i}<\min \left\{\delta_{r} / 100,1 / i\right\}$. Thus $\left(\tilde{B}\left(p_{j_{i}}, r_{j_{i}}, R_{i, j_{i}}\right)^{\delta_{i}}, \tilde{p}_{j_{i}}\right)$ converge to $\bar{B}(y, r)$ as well.

Furthermore, we claim that $\pi_{i}: \tilde{B}\left(p_{j_{i}}, r_{j_{i}}, R_{i, j_{i}}\right)^{\delta_{i}} \rightarrow B\left(p_{i}, r_{j_{i}}\right)$ is an isometry on balls of radius $\delta_{r} / 4$. If this were not true, then there would be a pair of lifts $\tilde{p}_{i}$ and $\tilde{p}_{i}^{\prime}$ of $p_{i}$ such that $d\left(\tilde{p}_{i}, \tilde{p}_{i}^{\prime}\right)<\delta_{r} / 2$. By the convergence of the covering maps in Theorem 2.9. and the fact that we are within $\epsilon_{i}$ of the limit, this implies that there are two lifts $\tilde{y}_{i}$ and $\tilde{y}_{i}^{\prime}$ of $y$ in $B\left(y, r_{i}, R_{i}\right)^{\delta_{i}}$, such that $d\left(\tilde{y}_{i}, \tilde{y}_{i}^{\prime}\right)<\delta_{r} / 2+2 \epsilon_{i}<\delta_{r}$. This contradicts the isometry of $\delta_{r} / 2$ balls on these relative delta covers.

Thus we can apply Proposition 3.8 with $R=\infty$, and $\delta=\delta_{r}$, to get a renormalized limit measure $\mu_{r}$ defined on $\bar{B}(y, r) \subset \tilde{Y}$ which satisfies the Bishop Gromov Volume Comparison for pairs of balls, $B\left(x, r_{1}\right), B\left(x, r_{2}\right)$, such that the radius of the inner ball satisfies $r_{1}<r-d_{Y}(\pi(x), y)$. Here the balls are measured using $d_{\bar{B}(y, r)}$ and the outer radius can be arbitrarily large. To get Bishop Gromov for balls defined using $d_{\tilde{Y}}$ we apply Lemma 2.2, and restrict the outer radius $r_{2}<r / 3-d_{Y}(\pi(x), y)$.

Furthermore, by Corollary 3.9 and Lemma 4.4, this renormalized limit measure agrees with the lifted measure $\mu_{\pi}$ as follows:

$$
\mu_{r}(S)=\lambda_{r} \mu_{\pi}(S)
$$

where

$$
\lambda_{r}=\lim _{i \rightarrow \infty} \frac{\operatorname{Vol}\left(B\left(p_{i}, 1\right) \subset M_{i}\right)}{\operatorname{Vol}\left(B\left(\tilde{p}_{i}, r / 10\right) \subset \tilde{B}\left(p_{j_{i}}, r_{j_{i}}, R_{i, j_{i}}\right)^{\delta_{i}}\right)} \in\left[\frac{V(n, H, \delta / 2)}{V(n, H, r / 10)}, \frac{V(n, H, 1)}{V(n, H, \delta / 2)}\right] .
$$

Since the Bishop Gromov Volume Comparison is a ratio, it also holds with respect to the lifted measure $\mu_{\pi}$ for balls measured with $d_{\tilde{Y}}$ of radius bounded by $r$ as above. However, this is true for any $r>0$, so Bishop Gromov holds for balls of all sizes and locations.

We also get a splitting theorem on $Y$ if the sequence of manifolds have Ricci curvature converging towards a nonnegative lower bound.

Theorem 4.6. Let $\left(M_{i}^{n}, p_{i}\right)$ be a sequence of manifolds with Ric Ri $_{M_{i}} \geq$ $-(n-1) \epsilon_{i}, \epsilon_{i} \rightarrow 0$, and converges to $(Y, y)$ in the pointed Gromov-Hausdorff sense. By Theorem 1.1, the universal cover of $Y, \tilde{Y}$, exists. If $\tilde{Y}$ contains a line, then $\tilde{Y}$ splits isometrically, $\tilde{Y}=\mathbb{R} \times \bar{X}$.

Proof. Let us assume the line of $\tilde{Y}$ passes through $\tilde{x} \in \tilde{Y}$. Given any $R>0, L>1$, let $B(\tilde{x}, 2 L R)$ be a ball in $\tilde{Y}$. Then there is a line segment $\tilde{\gamma}:(-2 L R, 2 L R) \rightarrow$ $B(\tilde{x}, 2 L R)$ with $\tilde{\gamma}(0)=\tilde{x}$. Let $x=\pi(\tilde{x})$ and $\bar{B}(x, 6 L R)$ be the connected lift of $B(x, 6 L R) \subset Y$ in $\tilde{Y}$ containing $\tilde{x}$. Then $B(\tilde{x}, 2 L R) \subset \tilde{Y}$ are the same as $B(\tilde{x}, 2 L R) \subset \bar{B}(x, 6 L R)$. By Corollary $4.3(\bar{B}(x, 6 L R), \tilde{x})$ is the pointed GromovHausdorff limit of a sequence of relative $\delta$-covers of manifolds with

$$
\operatorname{Ric} \geq-(n-1) \epsilon_{k},
$$


namely $\left(\tilde{B}\left(x_{k}, r_{k}, R_{k}\right)^{\delta_{k}}, \tilde{x}_{j_{i}}\right)$, where $r_{k} \rightarrow 6 L R$ and $R_{k} \rightarrow \infty$. Let $q_{k}^{+}, q_{k}^{-} \in$ $\tilde{B}\left(x_{k}, r_{k}, R_{k}\right)^{\delta_{k}}$ be the $\epsilon_{k}$ Hausdorff images of the points $\tilde{\gamma}(2 L R), \tilde{\gamma}(-2 L R)$. Now given any $\epsilon>0$, choose $L=L(n, \epsilon)$ to be the constant in ChCo1, Proposition 6.2]. Then $\min \left(d\left(x_{k}, q_{k}^{+}\right), d\left(x_{k}, q_{k}^{-}\right)\right) \geq L R$, the excess at $x_{k}$ with respect to $q_{k}^{+}, q_{k}^{-}$, $E\left(x_{k}\right) \leq 3 \epsilon_{k}$, since $\epsilon_{k} \rightarrow 0$ as $k \rightarrow \infty$. We have for all $k$ big, $E\left(x_{k}\right) \leq \tau R$ and Ric $\leq$ $-(n-1) \tau R^{-2}$. By [ChCo1, Theorem 6.62], the ball $B\left(x_{k}, R\right) \subset \tilde{B}\left(x_{k}, r_{k}, R_{k}\right)^{\overline{\delta_{k}}}$ is $\epsilon R$ Gromov-Hausdorff close to an $R$-ball in $\mathbb{R} \times X_{k}$, for some metric space $X_{k}$. Therefore the limit ball $B(\tilde{x}, R)$ is isometric to an $R$-ball in $\mathbb{R} \times X$, for some metric space $X$. This is true for any $R$ ball in $\tilde{Y}$. Hence $\tilde{Y}$ splits globally.

Using Theorem 4.5 we can easily extend several results about manifolds with nonnegative Ricci curvature to limit spaces.

First we can extend Milnor's result [Mi] about fundamental groups of polynomial growth to the revised fundamental groups of limit spaces.

Corollary 4.7. If $(Y, y)$ is a pointed Gromov-Hausdorff limit of a sequence of complete manifolds $\left(M_{i}^{n}, p_{i}\right)$ with Ric $M_{M_{i}} \geq-(n-1) \epsilon_{i}, \epsilon_{i} \rightarrow 0$, then any finitely generated subgroup of the revised fundamental group of $Y, \bar{\pi}_{1}(Y)$, is of polynomial growth of degree at most $n$.

We can also extend Anderson's Theorems from [An2], regarding volume growth and the revised fundamental group. In particular

Corollary 4.8. If $(Y, y)$ is a pointed Gromov-Hausdorff limit of a sequence of complete manifolds $\left(M_{i}^{n}, p_{i}\right)$ with Ric $M_{M_{i}} \geq 0$, and if it has Euclidean measure growth $\liminf _{r \rightarrow \infty} \mu\left(B_{\tilde{y}}(r)\right) / r^{n}=C>0$, then the revised fundamental group is finite and $\left|\bar{\pi}_{1}(Y, y)\right| \leq \omega_{n} / C$.

We will say that a length space $Y$ has the loops to infinity property if given any element $g$ of the revised fundamental group of $Y$ based at $y$ and given any compact set $K$ in $Y, g$ has a representative element of the form $\gamma \circ C \circ \gamma^{-1}$, where $C$ is a loop in $M \backslash K$ and $\gamma$ is a minimal curve running from $y$ to $M \backslash K$.

Using this definition it is easy to imitate the beginning of the proof in [So2] to obtain the following:

Corollary 4.9. If $M_{i}$ have Ricci $\left(M_{i}\right) \geq-\epsilon_{i}$ with $\epsilon_{i}$ decreasing to 0 , and if $Y=$ $G H \lim _{i \rightarrow \infty} M_{i}$, then either $Y$ has the loops to infinity property or the universal cover of $Y$ splits isometrically.

Finally we close with a theorem relating the local fundamental groups of the $M_{i}$ to the revised fundamental groups in $\mathrm{Y}$.

Proposition 4.10. $G\left(p_{i}, r_{i}, R_{i}\right)$ maps surjectively to $\mathrm{im}\left(\bar{\pi}_{1}(B(y, r), y) \rightarrow \bar{\pi}_{1}(Y, y)\right)$ for $i \geq N_{r, R, \delta}$, where $G\left(p_{i}, r_{i}, R_{i}\right)$ is the deck transformation of the connected lift of $B\left(p_{i}, r_{i}\right)$ in the universal cover of $B\left(p_{i}, R_{i}\right)$.

Recall Example 1.2 in which we showed that we cannot hope for surjectivity when we do not restrict our attention to balls. Recall also Example 4.1 which shows that even if $M_{i}=Y$, we can have a large kernel. Also, there is a sequence of $M_{i}$ converging to a cylinder $Y$ demonstrating that the kernel may be free even without collapsing. Just take $M_{i}=[-i, i] \times s^{\prime}$ with a pair of $T^{2} \backslash B(1)$ pasted smoothly to the circle $\{-i\} \times s^{\prime}$ and $\{i\} \times s^{\prime}$ along $\partial B(1)$. This is in strong contrast with the compact case in which the kernel is finite in the noncollapsed case. 
Proof. By Theorem 3.12 for any $r<R, \tilde{B}(y, r, R)^{\delta}$ stabilizes for $\delta<\delta_{y, r, R}$. So $G(y, r, R, \delta)$ also stabilizes for $\delta<\delta_{y, r, R}$. Call the stable group $G(y, r, R)$.

By Corollary 2.8 we have surjective maps from $G\left(p_{i}, r_{i}, R_{i}, \delta_{1}\right)$ to this stabilized group $G(y, r, R)$ for all $\delta_{1}<\delta_{y, r, R}$ and $i$ large. Now there are natural surjective maps from

$$
G\left(p_{i}, r_{i}, R_{i}\right)=i m\left(\pi_{1}\left(B\left(p_{i}, r_{i}\right), p_{i}\right) \rightarrow \pi_{1}\left(B\left(p_{i}, R_{i}\right), p_{i}\right)\right)
$$

onto $G\left(p_{i}, r_{i}, R_{i}, \delta_{1}\right)$. So we have surjective maps from $G\left(p_{i}, r_{i}, R_{i}\right)$ to $G(y, r, R)$ for $i \geq N_{r, R, \delta}$.

However $G(y, r, R)$ maps surjectively onto $i m\left(\bar{\pi}_{1}(B(y, r), y) \rightarrow \bar{\pi}_{1}(Y, y)\right)$ because $\tilde{B}(y, r, R)^{\delta_{y, r, R}}$ covers the connected lift of $B(y, r)$ in $\tilde{Y}$.

Thus $G\left(p_{i}, r_{i}, R_{i}\right)$ maps onto $i m\left(\bar{\pi}_{1}(B(y, r), y) \rightarrow \bar{\pi}_{1}(Y, y)\right)$ for $i \geq N_{r, R, \delta}$.

\section{Appendix}

Once and for all we show that the two possible definitions of Gromov Hausdorff convergence on noncompact spaces are identical.

Definition 5.1. We say $\left(M_{i}, p_{i}\right)$ converges in the pointed Gromov Hausdorff sense to $(Y, y)$ if for all $R>0,\left(B\left(p_{i}, R\right), d_{M_{i}}\right)$ converges to $\left(B(y, R), d_{Y}\right)$ in the Gromov Hausdorff sense.

This definition works well because if $M_{i}$ happens to have a uniform upper bound on diameter and $M_{i}$ converges to $Y$, then there exist $p_{i}$ and $y$ such that $\left(M_{i}, p_{i}\right)$ converges in the pointed Gromov Hausdorff sense to $(Y, y)$. See [Pe, Page 279].

On the other hand, Gromov emphasizes the importance of length spaces in his text. For our purposes it is essential to use length spaces. Thus there is another possible definition.

Definition 5.2. We say $\left(M_{i}, p_{i}\right)$ converges in the pointed Gromov Hausdorff sense to $(Y, y)$ if for all $R>0$, there exists $R_{i} \rightarrow R$ such that $\left(B\left(p_{i}, R_{i}\right), d_{B\left(p_{i}, R_{i}\right)}\right)$ converging to $\left(B(y, R), d_{B(y, R)}\right)$ in the Gromov Hausdorff sense.

Note that if $M_{i}$ converges to $X$ in Gromov-Hausdorff topology and $p_{i} \in M_{i}$ converges to $x \in X$, it may not be true that $B\left(p_{i}, R\right)$ in $M_{i}$ with intrinsic metric converges to $B(x, R)$ in $X$ with intrinsic metric. For example, let $M_{i}$ be circles of radius $1+(1 / i)$ converging to $X$, the circle with radius 1 . Then the balls of radius $\pi$ in $M_{i}$ with intrinsic metric is the interval $[0,2 \pi]$ while the ball of radius $\pi$ in $X$ is the unit circle. Therefore in the above one needs $R_{i}$, not just $R$.

Lemma 5.1. Definition 5.1 and Definition 5.2 are equivalent.

Before proving this statement, we make the following definition.

Definition 5.3. Given two metric spaces $X, Y$, a map $\varphi: X \rightarrow Y$ is said to be an $\epsilon$-Hausdorff approximation if the following conditions are satisfied:

1) The $\epsilon$-neighborhood of $\varphi(X)$ in $Y$ is equal to $Y$.

2) For each $x_{1}, x_{2}$ in $X$, we have

$$
\left|d_{X}\left(x_{1}, x_{2}\right)-d_{Y}\left(\varphi\left(x_{1}\right), \varphi\left(x_{2}\right)\right)\right| \leq \epsilon .
$$

We say metrics space $X_{i}$ converges to $X$ in Gromov-Hausdorff topology if there are $\epsilon_{i}$-Hausdorff approximations from $X_{i}$ to $X$ and $\epsilon_{i} \rightarrow 0$ as $i \rightarrow \infty$. 
Proof. We first show that if $\left(M_{i}, p_{i}\right)$ converges in the pointed Gromov Hausdorff sense to $(Y, y)$ according to Definition $[5.2$ then it converges according to Definition 5.1 as well.

For all $R>0$, we know there exists $\rho_{i} \rightarrow 3 R$ such that $\left(B\left(p_{i}, \rho_{i}\right), d_{B\left(p_{i}, \rho_{i}\right)}\right)$ converges to $\left(B(y, 3 R), d_{B(y, 3 R)}\right)$ in the Gromov Hausdorff sense. Therefore $\left(B\left(p_{i}, R\right), d_{B\left(p_{i}, \rho_{i}\right)}\right)$ converges to $\left(B(y, R), d_{B(y, 3 R)}\right)$ in the Gromov Hausdorff sense as well. However by Lemma 2.2 $\left(B\left(p_{i}, R\right), d_{B\left(p_{i}, \rho_{i}\right)}\right)$ is isometric to $\left(B\left(p_{i}, R\right), d_{M_{i}}\right)$ and $\left(B(y, R), d_{B(y, 3 R)}\right)$ is isometric to $\left(B(y, R), d_{Y}\right)$ and we are done.

The other direction is less trivial.

Assume $\left(M_{i}, p_{i}\right)$ converges in the pointed Gromov Hausdorff sense to $(Y, y)$ according to Definition 5.1. Fix $R>0$. We must construct $R_{i} \rightarrow R$.

First there exists $\epsilon_{i} \rightarrow 0$ such that

$$
d_{G H}\left(\left(B\left(p_{i}, 3 R\right), d_{M_{i}}\right),\left(B(y, 3 R), d_{Y}\right)\right)<\epsilon_{i}
$$

so there exists

$$
f_{i}: B(y, 3 R) \rightarrow B\left(p_{i}, 3 R\right) \text { where } f_{i}(y)=p_{i}
$$

which is an $\epsilon_{i}$-Hausdorff approximation with restricted metrics.

Now $f_{i}: B(y, R) \rightarrow B\left(p_{i}, R+\epsilon_{i}\right)$ and we would like to show that it is almost distance preserving and almost onto with the intrinsic distances. However, we will not be able to do so without adding a little extra space. So we will look at

$$
f_{i}: B(y, R) \rightarrow B\left(p_{i}, R+\epsilon_{i}+\delta_{i}\right)
$$

where $\delta_{i}=\sqrt{\epsilon_{i}}$.

We will show that $\forall \delta>0$ there exists $i$ sufficiently large such that $f_{i}: B(y, R) \rightarrow$ $B\left(p_{i}, R+\epsilon_{i}+\delta_{i}\right)$ are $\delta$-Hausdorff approximations with the intrinsic distances. Note that $f_{i}$ could be far from being such a map if $i$ is not taken sufficiently large. Take $Y=M_{i}=$ cylinders such that $B(y, R)$ does not have cut points but $B\left(p_{i}, R+\epsilon_{i}+\delta_{i}\right)$ does. Note that for $i$ sufficiently large $B\left(p_{i}, R+\epsilon_{i}+\delta_{i}\right)$ does not have cut points anymore.

We begin by showing that it is almost distance preserving. For all $a, b \in B(y, R)$ there is a curve $C \in B(y, R)$ from $a$ to $b$, such that $L(C)=d_{B(y, R)}(a, b) \leq 2 R$. We parametrize $C$ by arclength and take $t_{0}=0, t_{j}=t_{j-1}+\delta_{i}$ and $t_{N} \leq t_{N-1}+\delta_{i}$ so that $C\left(t_{0}\right)=a, C\left(t_{N}\right)=b$,

$$
d_{Y}\left(C\left(t_{j}\right), C\left(t_{j+1}\right)\right) \leq d_{B(y, R)}\left(C\left(t_{j}\right), C\left(t_{j+1}\right)\right) \leq \delta_{i}
$$

and

$$
L(C)-\delta_{i} \leq(N-1) \delta_{i} \leq L(C) .
$$

Now $f_{i}(a)$ and $f_{i}(b)$ are joined by a curve $C_{i}$ which is created by joining the points $f_{i}\left(t_{j}\right)$ by minimal curves in $M_{i}$. Note that then $C_{i} \subset B\left(p_{i}, R+\epsilon_{i}+\frac{\epsilon_{i}+\delta_{i}}{2}\right)$, 
and

$$
\begin{aligned}
L\left(C_{i}\right) & =\sum_{j=1}^{N} d_{M_{i}}\left(f_{i}\left(C\left(t_{j-1}\right)\right), f_{i}\left(C\left(t_{j}\right)\right)\right) \\
& \leq \sum_{j=1}^{N}\left(d_{Y}\left(C\left(t_{j-1}\right), C\left(t_{j}\right)\right)+\epsilon_{i}\right) \\
& \leq N\left(\delta_{i}+\epsilon_{i}\right)=N \delta_{i}\left(1+\delta_{i}\right) \\
& \leq\left(L(C)+\delta_{i}\right)\left(1+\delta_{i}\right) \leq L(C)+(2 R+1) \delta_{i}+\epsilon_{i} .
\end{aligned}
$$

Thus

$$
d_{B\left(p_{i}, R+\epsilon_{i}+\delta_{i}\right)}\left(f_{i}(a), f_{i}(b)\right) \leq d_{B(y, R)}(a, b)+(2 R+1) \delta_{i}+\epsilon_{i} .
$$

On the other hand, $d_{B\left(p_{i}, R+\epsilon_{i}+\delta_{i}\right)}\left(f_{i}(a), f_{i}(b)\right)=L\left(\sigma_{i}\right)=L_{i}$ for some curve $\sigma_{i}:\left[0, L_{i}\right] \rightarrow B\left(p_{i}, R+\epsilon_{i}+\delta_{i}\right)$ connecting $f_{i}(a), f_{i}(b)$. Let $L_{\infty}=\liminf _{i \rightarrow \infty} L_{i}$. Then there is a subsequence converging to $L_{\infty}$. Since $\left(B\left(p_{i}, R+\epsilon_{i}+\delta_{i}\right), d_{M_{i}}\right)$ converges to $\left(B(y, R), d_{Y}\right)$, by the generalized Arzela-Ascoli theorem [GP], [Pe] Page 279, Lemma 1.8], there is a subsequence such that $\sigma_{i}$ converges to $\sigma_{\infty}$ : $\left[0, L_{\infty}\right] \rightarrow B(y, R)$ and $\sigma_{\infty}(0)=a$ and $\sigma_{\infty}\left(L_{\infty}\right)=b$.

Thus $d_{B(y, R)}(a, b) \leq L\left(\sigma_{\infty}\right)=\lim _{i \rightarrow \infty} L\left(\sigma_{i}\right)=L_{\infty}$. Since $L_{\infty}=\liminf _{i \rightarrow \infty} L_{i}$, for all $\delta>0$ there exists $N_{a, b, \delta}$ sufficiently large such that

$$
d_{B(y, R)}(a, b) \leq d_{B\left(p_{i}, R+\epsilon_{i}+\delta_{i}\right)}\left(f_{i}(a), f_{i}(b)\right)+\delta \quad \forall i \geq N_{a, b, \delta} .
$$

However we need a uniform estimate for $N$ not depending on $a$ and $b$ to say that $f_{i}$ is almost distance preserving.

We assume on the contrary that there exists $\delta>0$ and a subsequence $i \rightarrow \infty$ and points $a_{i}, b_{i} \in B(y, R)$ such that

$$
d_{B(y, R)}\left(a_{i}, b_{i}\right) \geq d_{B\left(p_{i}, R+\epsilon_{i}+\delta_{i}\right)}\left(f_{i}\left(a_{i}\right), f_{i}\left(b_{i}\right)\right)+\delta .
$$

Then a subsequence of $a_{i}$ and of $b_{i}$ converge to points $a$ and $b$ in $B(y, R)$ for which (5.11) holds. So take $i \geq N_{a, b, \delta / 10}$ and also large enough that $(2 R+1) \delta_{i}+\epsilon_{i}<\delta / 2$, $d_{B(y, R)}\left(a, a_{i}\right)<\delta / 10$ and $d_{B(y, R)}\left(b, b_{i}\right)<\delta / 10$. Then we can apply (5.11) and (5.10) to get

$$
\begin{aligned}
d_{B(y, R)}\left(a_{i}, b_{i}\right) & \leq 2(\delta / 10)+d_{B(y, R)}(a, b) \\
& \leq 3(\delta / 10)+d_{B\left(p_{i}, R+\epsilon_{i}+\delta_{i}\right)}\left(f_{i}(a), f_{i}(b)\right) \\
& \leq 3(\delta / 10)+d_{B(y, R)}(a, b)+(2 R+1) \delta_{i}+\epsilon_{i}<d_{B(y, R)}(a, b)+\delta,
\end{aligned}
$$

which contradicts (5.12).

Thus (5.11) holds without the dependence on $a$ and $b$. Combining this with (5.10) we have, $\forall \delta>0, \exists N_{\delta}$ such that $f_{i}$ is $\delta$ almost distance preserving for all $i \geq N_{\delta}$.

Now to prove that $f_{i}$ is $\delta$ almost onto, we note that

(5.16) $\forall q \in B\left(p_{i}, R+\epsilon_{i}+\delta_{i}\right) \subset B\left(p_{i}, 3 R\right) \quad \exists z_{q}^{\prime} \in B(y, 3 R)$ s.t. $d_{M_{i}}\left(f_{i}\left(z_{q}^{\prime}\right), q\right)<\epsilon_{i}$.

If $q \in B\left(p_{i}, R-2 \epsilon_{i}\right)$, then

$$
\begin{aligned}
d_{Y}\left(z_{q}^{\prime}, y\right) & \leq d_{M_{i}}\left(f_{i}\left(z^{\prime}\right), p_{i}\right)+\epsilon_{i} \\
& \leq d_{M_{i}}\left(q, p_{i}\right)+d_{M_{i}}\left(f_{i}\left(z^{\prime}\right), q\right)+\epsilon_{i}<R-2 \epsilon_{i}+2 \epsilon_{i} .
\end{aligned}
$$


So $z_{q}^{\prime} \in B(y, R)$. Furthermore the minimal curve from $f_{i}\left(z_{q}^{\prime}\right)$ to $q$ must be in $B\left(p_{i}, R-\epsilon_{i}\right)$ so

$$
d_{B\left(p_{i}, R+\epsilon_{i}+\delta_{i}\right)}\left(z_{q}^{\prime}, q\right)=d_{M_{i}}\left(z_{q}^{\prime}, q\right)<\epsilon_{i} .
$$

If $q \in B\left(p_{i}, R+\epsilon_{i}+\delta_{i}\right)$, then let $\bar{q}$ be the first point on a minimal geodesic joining $q$ to $p_{i}$ which is in $B\left(p_{i}, R-2 \epsilon_{i}\right)$. So $d_{B\left(p_{i}, R+\epsilon_{i}+\delta_{i}\right)}(q, \bar{q})<3 \epsilon_{i}+\delta_{i}$. Then let $z_{q}=z_{\bar{q}}^{\prime}$, so

$$
\begin{aligned}
d_{B\left(p_{i}, R+\epsilon_{i}+\delta_{i}\right)}\left(z_{q}, q\right) & \leq d_{B\left(p_{i}, R+\epsilon_{i}+\delta_{i}\right)}(q, \bar{q})+d_{B\left(p_{i}, R+\epsilon_{i}+\delta_{i}\right)}\left(z_{q}, \bar{q}\right) \\
& <3 \epsilon_{i}+\delta_{i}+\epsilon_{i} .
\end{aligned}
$$

Thus $f_{i}$ is $4 \epsilon_{i}+\delta_{i}$ almost onto with respect to the intrinsic distances.

Since $4 \epsilon_{i}+\delta_{i}$ converges to 0 , we are done.

Corollary 5.2. If $\left(M_{i}, p_{i}\right)$ converges in the pointed Gromov Hausdorff sense to $(Y, y)$, then for any $0<r<R$, there exist $r_{i} \rightarrow r, R_{i} \rightarrow R$, such that for all $\delta>0$ there exists $N_{\delta}(r, R)$ and maps $f_{i}$ such that for all $i \geq N_{\delta}(r, R)$ the maps $f_{i}$ are $\delta$ Hausdorff approximations $f_{i}: B(y, R) \rightarrow B\left(p_{i}, R_{i}\right)$ with respect to intrinsic metrics, and their restrictions $f_{i}: B(y, r) \rightarrow B\left(p_{i}, r_{i}\right)$ are also $\delta$-Hausdorff approximation with respect to the intrinsic distances on these smaller balls.

\section{REFERENCES}

[AbGl] U. Abresch, D. Gromoll, On complete manifolds with nonnegative Ricci curvature, J. Amer. Math. Soc. 3 (1990) 355-374. MR 91a:53071

[An1] M. T. Anderson Convergence and rigidity of manifolds under Ricci curvature bounds, Invent. Math. 102 (1990), no. 2, 429-445. MR 92c:53024

[An2] M. Anderson, On the topology of complete manifolds of non-negative Ricci curvature, Topology 29 (1990), no. 1, 41-55. MR 91b:53041

[BiCr] R. Bishop, R. Crittenden, Geometry of manifolds. Reprint of the 1964 original. AMS Chelsea Publishing, Providence, RI, 2001. MR 2002d:53001

[BoMe] B.H. Bowditch, G. Mess, A 4-dimensional Kleinian group. Trans. Amer. Math. Soc. 344 (1994), no. 1, 391-405. MR 95f:57057

[BBI] D. Burago, Y. Burago, S. Ivanov, A course in Metric Geometry. Graduate Studies in Mathematics, Vol. 33, AMS, 2001. MR 2002e:53053

[ChCo1] J. Cheeger, T. Colding, Lower bounds on Ricci curvature and almost rigidity of warped products, Ann. of Math. (2) 144 (1996), no. 1, 189-237. MR 97h:53038

[ChCo2] J. Cheeger, T. Colding, On the structure of spaces with Ricci curvature bounded below I, J. Diff. Geom. 46 (1997) 406-480. MR 98k:53044

[ChCo3] J. Cheeger, T. Colding, On the structure of spaces with Ricci curvature bounded below II, J. Differential Geom. 54 (2000), no. 1, 13-35. MR 2003a:53043

[ChCo4] J. Cheeger, T. Colding, On the structure of spaces with Ricci curvature bounded below III, J. Differential Geom. 54 (2000), no. 1, 37-74. MR 2003a:53044

[Fed] H. Federer, Geometric Measure Theory, Springer, 1969. MR 41:1976

[Gr] M. Gromov, Metric structures for Riemannian and non-Riemannian spaces, PM 152, Birkhauser, 1999. MR 2000d:53065

[GP] K. Grove, P. Petersen, Manifolds near the boundary of existence, J. Diff. Geom. 33 (1991) 379-394. MR 92a:53067

[Ma] W. Massey, A basic course in algebraic topology, GTM 127, Springer-Verlag, 1991. MR 92c:55001

[Me] X. Menguy, Examples with bounded diameter growth and infinite topological type. Duke Math. J. 102 (2000), no. 3, 403-412. MR 2001c:53041

[Mi] J. Milnor, A note on curvature and fundamental group, J. Diff. Geom. 2 (1968) 1-7. MR 38:636

[Mun] M. E. Munroe, Introduction to measure and integration. Addison-Wesley Publishing Company, Inc., Cambridge, Mass., 1953. MR 14:734a 
[Nab] P. Nabonnand, Sur les variétés riemanniennes complètes à courbure de Ricci positive, C. R. Acad. Sci. Paris Sér. A-B 291 (1980), 591-593. MR 81m:53054

[Pl1] G. Perelman, The beginning of the Morse theory on Alexandrov spaces, St. Petersburg Math. Journ. 5 (1994), no. 1, 205-214. MR 94h:53054

[P12] G. Perelman, Construction of manifolds of positive Ricci curvature with big volume and large Betti numbers, in "Comparison Geometry" (Berkeley, CA, 1993-94), 157-163. MR 98h:53062

[Pe] P. Petersen, Riemannian geometry, GTM 171, Springer-Verlag, 1998. MR 98m:53001

[Po] L. Potyagailo, Finitely generated Kleinian groups in 3-space and 3-manifolds of infinite homotopy type, Trans. Amer. Math. Soc. 344 (1994), no. 1, 57-77. MR 94m:57030

[So1] C. Sormani, Nonnegative Ricci curvature, small linear diameter growth and finite generation of fundamental groups, Journal of Differential Geometry 54 (2000) 547-559. MR 2003a:53047

[So2] C. Sormani, On Loops Representing Elements of the Fundamental Group of a Complete Manifold with Nonnegative Ricci Curvature, Indiana Univ. Math. J. 50 (2001), no. 4, 1867-1883. MR 2003b:53038

[SoWei] C. Sormani and G. Wei, Hausdorff Convergence and Universal Covers, Transactions of the American Mathematical Society 353 (2001) 3585-3602. MR 2002e:53057

[Sp] E. Spanier, Algebraic Topology, McGraw-Hill, Inc., 1966. MR 35:1007

[Wei] G. Wei, Examples of complete manifolds of positive Ricci curvature with nilpotent isometry groups, Bull. Amer. Math. Soci. Vol. 19, no. 1 (1988), 311-313. MR 89h:53101

Department of Mathematics and Computer Science, Lehman College, City University of New York, Bronx, New York 10468

E-mail address: sormani@g230.lehman.cuny.edu

Department of Mathematics, University of California, Santa Barbara, California 93106

E-mail address: wei@math.ucsb.edu 